\title{
UNIFORM CONTROL OF LOCAL TIMES OF SPECTRALLY POSITIVE STABLE PROCESSES ${ }^{1}$
}

\author{
By NOAH FORMAN * , SOUMIK PAL*, DOUGlas RizzOlO ${ }^{\dagger}$ \\ AND MATTHIAS WINKEL \\ University of Washington, ${ }^{*}$ University of Delaware ${ }^{\dagger}$ and University of Oxford ${ }^{\ddagger}$
}

We establish two results about local times of spectrally positive stable processes. The first is a general approximation result, uniform in space and on compact time intervals, in a model where each jump of the stable process may be marked by a random path. The second gives moment control on the Hölder constant of the local times, uniformly across a compact spatial interval and in certain random time intervals. For the latter, we introduce the notion of a Lévy process restricted to a compact interval, which is a variation of Lambert's Lévy process confined in a finite interval and of Pistorius' doubly reflected process. We use the results of this paper to exhibit a class of path-continuous branching processes of Crump-Mode-Jagers-type with continuum genealogical structure. A further motivation for this study lies in the construction of diffusion processes in spaces of interval partitions and $\mathbb{R}$ trees, which we explore in forthcoming articles. In that context, local times correspond to branch lengths.

1. Introduction. Consider a spectrally positive Lévy process $X=\left(X_{t}, t \geq 0\right)$ with Laplace exponent $\psi(\eta)=\eta^{1+\alpha}$ and Lévy measure $\Pi(d x)=(1+\alpha) \alpha(\Gamma(1-$ $\alpha))^{-1} x^{-\alpha-2} d x$, for some $\alpha \in(0,1)$. We mark each jump $\left(t, \Delta X_{t}\right)$ independently by a continuous random nonnegative path $Z_{t}=\left(Z_{t}(s), 0 \leq s \leq \Delta X_{t}\right)$ with $Z_{t}(0)=Z_{t}\left(\Delta X_{t}\right)=0$, using a marking kernel $x \mapsto \kappa_{q}(x, \cdot)$ that has the following self-similarity property for some $q>0$. If $Z$ has distribution $\kappa_{q}(1, \cdot)$, then $\left(x^{q} Z(s / x), 0 \leq s \leq x\right)$ has distribution $\kappa_{q}(x, \cdot)$. We consider the levels in the jump interval $\left[X_{t-}, X_{t}\right]$ as the (vertical) time interval of the path, associating $Z_{t}(s)$ with level $X_{t-}+s$. If we interpret each $Z_{t}(s)$ as a population size or mass at level $X_{t-}+s$, the aggregate at time $y$ associated with $\left(X_{t}, 0 \leq t \leq T\right)$ is given by

$$
Z_{[0, T]}(y)=\sum_{0 \leq t \leq T} Z_{t}\left(y-X_{t-}\right), \quad y \in \mathbb{R}, T \geq 0,
$$

with the convention that $Z_{t}(s)=0$ for $s \notin\left(0, \Delta X_{t}\right)$.

Received October 2016; revised August 2017.

${ }^{1}$ Supported in part by NSF Grants DMS-12-04840, DMS-13-08340, DMS-16-12483, UW-RRF Grant A112251, EPSRC Grant EP/K029797/1 and the University of Delaware Research Foundation. MSC2010 subject classifications. 60G51, 60G52, 60J55, $60 \mathrm{~J} 80$.

Key words and phrases. Stable process, squared Bessel processes, CMJ process, local time approximation, excursion theory, restricted Lévy process, Hölder continuity. 
Structurally, (1) is the same as the sum of characteristics in a Crump-ModeJagers (CMJ) model. In a CMJ model of a population, individuals survive for a random amount of time and give birth to offspring at random times during their lifetime. The offspring of an individual then evolve independently in the same way. Moreover, each individual is equipped with a characteristic that can be observed over time. For example, the characteristic of the individual $i$ at age $s$ could be the indicator of whether or not individual's lifetime exceeds $s$ or it could be the fitness of the individual when its age is $s$. In the original work on this model, Jagers [24, 25 ] considers the process

$$
C_{y}=\sum_{i} \chi_{i}\left(y-\sigma_{i}\right), \quad y \geq 0,
$$

where the sum is over individuals $i$ that ever exist and individual $i$ has birth time $\sigma_{i}$, lifetime $\lambda_{i}$ and characteristic $\chi_{i}$, which is a function $\chi_{i}: \mathbb{R} \rightarrow \mathbb{R}$ whose support is contained in $\left[0, \lambda_{i}\right]$. The correspondence between $\left(i, \lambda_{i}, \sigma_{i}\right)$ in (2) and $\left(t_{i}, \Delta X_{t_{i}}, X_{t_{i}-}\right)$ in (1) is due to Lambert [31], who constructed $X$ as a boundedvariation Lévy process, in the binary homogeneous case, where the $\sigma_{i}$ are distinct and individuals give birth according to a Poisson process during their lifetimes.

Suitably rescaled, these Lévy processes converge to a spectrally positive stable process, which is given a genealogical interpretation in [33], and [32] shows that the rescaled number of individuals in the population converges to the local time of the Lévy process. Our formula (1) is the analogue for these Lévy processes of the sum of characteristics in a CMJ model. In Theorem 1 below, we show that the local time of the Lévy process can be recovered from the characteristics. We remark that Lévy processes marked by Poisson processes were studied by Delaporte $[13,14]$.

Our results also have a population genetics interpretation due to a connection with infinitely-many-neutral-alleles diffusion models of Ethier and Kurtz [17] and Petrov [37]; see [19]. In this setting, which we adopt for the remainder of this paper, the fundamental quantities of interest are genetic types and the important characteristic of a genetic type is its frequency. By thinking of the members of a population with the same genetic type in aggregate as an "individual" which gives birth to new genetic types via a birth mutation mechanism, one natural way to model the genetic structure of a population is to use a CMJ model in which "individuals" are genetic types and "characteristics" are type frequencies. Using this correspondence, the number of individuals can be seen as a notion of diversity of the population, varying as a function of time.

This notion, where each nonzero part in the sum (2) corresponds to an extant genetic type, can be extended to models (1) with infinitely many types by taking suitable scaling limits. Specifically, popular models for type frequencies include the two-parameter family of $\left(P_{i}, i \geq 1\right) \sim \operatorname{GEM}(\beta, \theta)$, also ranked as $\left(P_{i}^{\downarrow}, i \geq 1\right) \sim \operatorname{PD}(\beta, \theta)$, and associated partition-valued Chinese Restaurant Processes $\left(\Pi_{n}, n \geq 1\right)$ obtained by sampling from frequencies $\left(P_{i}, i \geq 1\right)$ and recording the types partitions $\Pi_{n}$ with $K_{n}=\# \Pi_{n}$ types among the first $n$ samples. In this 
framework, the $\beta$-diversity can be defined by any of the following limits:

$$
\begin{aligned}
S_{\beta} & =\lim _{n \rightarrow \infty} \frac{K_{n}}{n^{\beta}}=\Gamma(1-\beta) \lim _{i \rightarrow \infty} i\left(P_{i}^{\downarrow}\right)^{\beta} \\
& =\Gamma(1-\beta) \lim _{h \downarrow 0} h^{\beta} \#\left\{i \geq 1: P_{i} \geq h\right\} ;
\end{aligned}
$$

see, for example, [40], Definition 3.10 and Theorem 3.13, and [21], Theorem 2.1. In the present paper, we will study the last of these limits in the model (1), with sizes $\left(Z_{t}\left(y-X_{t-}\right), 0 \leq t \leq T\right)$ in the place of $\left(P_{i}, i \geq 1\right)$, and we will see that this yields approximations of the local times of $X$.

Boylan [9] showed that $X$ possesses jointly continuous local times $\left(\ell^{y}(t), y \in\right.$ $\mathbb{R}, t \geq 0)$. Barlow, Perkins and Taylor [2,3] established several uniform approximations of local times. Our first goal in this paper is to give a new intrinsic approximation of local times $\ell^{y}(t)$ using the marks at level $y$. We define for all $h>0$, $y \in \mathbb{R}$ and $T \geq 0$,

$$
\begin{aligned}
m_{h}(y, T) & =\sum_{0 \leq t \leq T} 1\left\{Z_{t}\left(y-X_{t-}\right) \geq h\right\} \\
& =\#\left\{0 \leq t \leq T: Z_{t}\left(y-X_{t-}\right) \geq h\right\} .
\end{aligned}
$$

This sum can be seen as a sum over excursions of $X$ away from level $y$. Since $X$ is spectrally positive, each such excursion has (at most) one jump across level $y$, so each excursion contributes just one term to the sum.

THEOREM 1. Let $\alpha \in(0,1)$ and $q>\alpha$. Suppose that $Z \sim \kappa_{q}(1, \cdot)$ is nontrivial in the sense that $\mathbb{P}(Z \equiv 0)<1$ and $\gamma$-Hölder continuous for some $\gamma \in(0, q)$, and that the Hölder constant

$$
D_{\gamma}:=\sup _{0 \leq r<s \leq 1} \frac{|Z(s)-Z(r)|}{|s-r|^{\gamma}}
$$

has moments of all orders. Then $c:=(1+\alpha)(\Gamma(1-\alpha))^{-1} \mathbb{E}\left((Z(U))^{\alpha / q}\right)>0$, where $U$ is a uniform variable on $[0,1]$ that is independent of $Z$, and the following holds almost surely for a $\kappa_{q}$-marked spectrally positive stable Lévy process $X$ with Laplace exponent $\psi(\eta)=\eta^{1+\alpha}$ :

$$
\lim _{h \downarrow 0} \sup _{0 \leq t \leq T} \sup _{y \in \mathbb{R}}\left|\frac{h^{\alpha / q}}{c} m_{h}(y, t)-\ell^{y}(t)\right|=0 \quad \text { for all } T>0 .
$$

To prove this result, we use excursion theory. Some steps resemble arguments of Khoshnevisan [27], who established rates of convergence of certain uniform approximations of the local times of Brownian motion. While we also obtain some rate of convergence results (Proposition 16) our arguments differ in several steps and do not appear to give rates of convergence for Theorem 1. 
The case where $\kappa_{q}$ gives squared Bessel bridges/excursions of dimension $\delta \in \mathbb{R}$ seems most compelling, in view of the branching structure (with immigration or emigration) of these processes that make them natural models for population size evolution, and in view of the Ray-Knight theorems. Specifically, the cases $\delta=0$ and $\delta=2$ appear in Ray-Knight theorems as distributions of Brownian local time as a process in the spatial variable. Generalisations to perturbed Brownian motions include other parameters; see, for example, [36, 43]. Squared Bessel processes and their excursions and bridges have self-similarity parameter $q=1$. Hölder continuity of these processes is well known, and we show in Corollary 36 that the moment assumption of Theorem 1 also holds for $\gamma \in(0,1 / 2)$. We restate this special case of Theorem 1 as Theorem 37.

Other interesting paths include some that are deterministic functions of the jump height, so that marking does not introduce additional randomness. The following example is obtained for deterministic $z(s)=s \wedge(1-s), 0 \leq s \leq 1$, and $\kappa(1, \cdot)=$ $\delta_{z}$, using $q=1$ to scale $z$ to $z_{x}(s)=s \wedge(x-s), 0 \leq s \leq x$ and $\kappa(x, \cdot)=\delta_{z_{x}}$ :

COROLLARY 2. Consider a spectrally positive stable process of index $1+\alpha \in$ $(1,2)$ and let

$$
m_{h}^{\circ}(y, T)=\#\left\{0 \leq t \leq T: X_{t-}+h \leq y \leq X_{t}-h\right\}, \quad h>0, y \in \mathbb{R}, T \geq 0 .
$$

Let $c^{\circ}=2^{\alpha} \Gamma(1-\alpha)$. Then the following holds almost surely for all $T>0$ :

$$
\lim _{h \downarrow 0} \sup _{0 \leq t \leq T} \sup _{y \in \mathbb{R}}\left|c^{\circ} h^{\alpha} m_{h}^{\circ}(y, t)-\ell^{y}(t)\right|=0 .
$$

This is clearly related to approximations by counting upcrossings [11, 27] or by jump heights. However, while we could obtain the latter by setting $z(s)=1$, this violation of the continuity requirement on $z$ seriously affects our arguments. The former cannot be expressed in this form, since whether an excursion contains an upcrossing depends not only on the single jump across the level, but on the preor post-jump parts of the excursion, as an upcrossing may be achieved by multiple upward jumps.

Let us turn to our second main result. We denote by $\tau^{y}(s)=\inf \left\{t \geq 0: \ell^{y}(t)>\right.$ $s\}$ the inverse local time of $X$ in level $y \in \mathbb{R}$. It is a well-known consequence of the scaling property of $X$ that $\tau^{0}$ is a stable subordinator, and hence has infinite mean. To obtain the following result, we restrict $X$ to space $[0, b]$ by making jumps across $b$ stop short at $b$, by excluding excursions above $b$ and below 0 , and similarly treating jumps from below 0 . Similar processes have been studied by Lambert [30], Pistorius [38] and indeed very recently Lambert and Uribe Bravo [33] proceeded similarly by restricting just at the upper boundary. See Section 5 for a precise discussion. We can state the theorem without this notion of a restricted Lévy process. 
THEOREM 3. Let $\alpha \in(0,1), b>0, v \in(0, \alpha / 2)$ and $S$ a random variable with moments of all orders. Then the local times of a spectrally positive stable process of index $1+\alpha$ have a uniform Hölder constant

$$
D_{\nu}^{[0, b]}\left(\tau^{0}(S)\right):=\sup _{0 \leq t \leq \tau^{0}(S)} \sup _{0 \leq x<y \leq b} \frac{\left|\ell^{y}(t)-\ell^{x}(t)\right|}{|y-x|^{v}}
$$

with moments of all orders.

Uniform Hölder continuity of local times was already obtained by Boylan [9]. The novelty of our result is in the finiteness of moments of the Hölder constant, which appears to be a fundamental fact about Lévy processes. The finiteness of Hölder constants is useful when dealing with families of scaled independent copies. We will apply Theorem 3 in this way in forthcoming work.

In Proposition 5, we provide a less technical illustration of this idea in the setting of Theorem 1, where Hölder constants of the $\kappa_{q}$-marks of the stable Lévy process have moments of all orders. Specifically, we devote the remainder of this introduction to the exploration of some auxiliary results, which may also be of independent interest. In particular, we will apply some of the auxiliary results to study some properties of the process $(y, t) \mapsto Z_{[0, t]}(y)$ of (1).

Consider the Itô excursion measure $n$ of $X$ away from 0 . Denote by $A$ and $B$ the undershoot and overshoot of the unique jump across level 0 under $n$, by $H=A+B$ the jump size and by $U=A / H$ the relative undershoot. The classical study of excursions away from 0 was recently complemented by Pardo et al. [34]. In the stable case, we can be slightly more explicit and deduce the following result. Symmetry under time-reversal is due to Getoor and Sharpe [20].

Proposition 4. We have $n(A \in d y, B \in d z)=(1+\alpha) \alpha(\Gamma(1-\alpha))^{-1}(y+$ $z)^{-\alpha-2} d z d y$ and $n(H \in d x, U \in d u)=(1+\alpha) \alpha(\Gamma(1-\alpha))^{-1} x^{-\alpha-1} d x d u$. In particular, under $n(\cdot \mid H=x), U$ is uniform on $[0,1]$. Under $n(\cdot \mid A=y, B=z)$, the pre-and post-jump parts of the excursion are independent. The post-jump part is a stable process starting from $z$ until hitting 0 . The pre-jump part is the negative of the time-reversal of a stable process starting from y until hitting 0.

Recall that Theorem 1 assumes the existence of moments for the Hölder constants of the random paths under $\kappa_{q}(1, \cdot)$. The $\kappa_{q}$-marked stable process contains a countably infinite collection of scaled independent copies of this random path. We can use the moments on the Hölder constants assumed in Theorem 1 in a simple Poisson sum over jumps to obtain uniform Hölder constants (not with all moments since the stable Lévy measure has a heavy tail).

PROPOSITION 5. In the setting of Theorem 1, the uniform Hölder constant for all paths $Z_{t}$ is finite a.s.: for all $T>0$,

$$
D_{[0, T]}:=\sup _{0 \leq t \leq T} \sup _{0 \leq x<y \leq \Delta X_{t}} \frac{\left|Z_{t}(y)-Z_{t}(x)\right|}{|y-x|^{\gamma}}<\infty .
$$


This is remarkable, since the unbounded variation of $X$ means that the jump heights are not summable, hence the time intervals of the processes $Z_{t}, 0 \leq t \leq T$, add up to infinite length. We can further improve on this proposition, as follows, to facilitate the aggregation (1) of mass at level $y$, for all levels $y \in \mathbb{R}$.

Proposition 6. In the setting of Theorem 1 , let $\gamma<q-\alpha$. Then the set $\{t \in$ $\left.[0, T]: \Delta X_{t}>0\right\}$ of jump times may a.s. be partitioned into sequences $\left\{J_{j}^{k}, j \geq 1\right\}$, $k \geq 1$, in such a way that:

(a) the jump intervals $\left[X\left(J_{j}^{k}-\right), X\left(J_{j}^{k}\right)\right], j \geq 1$, are disjoint for each $k \geq 1$;

(b) Hölder constants $D_{[0, T]}^{k}=\sup _{j \geq 1} \sup _{0 \leq x<y \leq \Delta X\left(J_{j}^{k}\right)} \frac{\left|Z_{J_{j}^{k}}(y)-Z_{J_{j}^{k}}(x)\right|}{|y-x|^{\gamma}}$ are summable in $k \geq 1$.

Let us return to the aggregate process (1) of Crump-Mode-Jagers-type. We begin by noting some properties that illustrate the genealogical complexity. Jagers' [24] framework is very general, but only covers branching processes with a discrete genealogy that is represented by a subset of the infinite Ulam-Harris tree $\mathbb{U}=$ $\bigcup_{n \geq 0} \mathbb{N}^{n}$. Lambert [31] used this framework to encode a class of CMJ processes in bounded-variation Lévy processes with negative drift, in such a way that the jumps correspond to the members of $\mathbb{U}$. Since all jumps happen at local minima, there is a unique previous jump across that local minimum level that corresponds to the parent. From any given jump, this yields a finite number of ancestors down to level 0 .

In our framework, $X$ has unbounded variation, no jumps occur from local minima (see, e.g., [4], Chapter VII), hence no direct parent for any jump can be identified in this way, but a collection of ancestral intervals and a continuum of limit levels between these can be put together to a continuum genealogy. This is very delicate and properly addressed in [33]. By marking the jumps of the stable process, we have bypassed the subtleties of the continuum genealogy and set up $Z_{[0, T]}$ directly building on the jump structure of the stable process, which by [33] allows the CMJ-type interpretation.

The results and methods we have discussed yield some properties of $Z_{[0, T]}$, while an exhaustive study of this interesting new class of processes is beyond the scope of the present paper.

COROLLARY 7. In the setting of Theorem 1 with $\gamma<q-\alpha$, the process $y \mapsto$ $Z_{[0, T]}(y)$ of (1) is a.s. $\gamma$-Hölder.

Recall that excursions of $X$ away from level $y$ have (at most) one jump across level $y$, while local time $\ell^{y}$ remains constant during each excursion. Therefore, inverse local time $\tau^{y}$ is a natural time scale for processes $t \mapsto m_{h}(y, t)$, and indeed for $t \mapsto Z_{[0, t]}(y)$. 
PROPOSITION 8. In the setting of Theorem 1 , we have for each $y \in \mathbb{R}$ the following subordinators:

(i) The process $s \mapsto Z_{\left[0, \tau^{y}(s)\right]}(y)-Z_{\left[0, \tau^{y}(0)\right]}(y)$ is a stable subordinator with index $\alpha / q$. Its Laplace exponent is $\Theta(\xi)=c \Gamma(1-\alpha / q) \xi^{\alpha / q}$.

(ii) The process $s \mapsto m_{h}\left(y, \tau^{y}(s)\right)-m_{h}\left(y, \tau^{y}(0)\right)$ is a Poisson process of rate $c h^{-\alpha / q}$ independent of $m_{h}\left(y, \tau^{y}(0)\right)=1\left\{Z_{T_{\geq y}}\left(y-X_{T_{\geq y}-}\right)>h\right\}$, where $T_{\geq y}=$ $\inf \left\{t>0: X_{t} \geq y\right\}$.

In particular, we deduce from (i) that $\left(Z_{\left[0, \tau^{y}(s)\right]}(y), 0 \leq s<\ell^{y}(T)\right)$ is a stopped stable subordinator. It is well known $[40,42]$ that its ranked relative jump sizes have Poisson-Dirichlet distribution, and that its $\beta$-diversity (for $\beta=\alpha / q$ ) can be approximated by the number of jumps/blocks exceeding threshold $h$, that is, by $m_{h}(y, T)$. Hence, Theorem 1 shows that this $\beta$-diversity process coincides with the local time process of $X$, and Theorem 3 is a statement on the Hölder-continuity of the $\beta$-diversity process as a function of $y$, expressing the evolution of the diversity of subpopulations $Z_{t}\left(y-X_{t-}\right), 0 \leq t \leq T$, in $Z_{[0, T]}(y)$. See Ruggiero et al. [45] for another recent study that exhibits continuous diversity processes.

A further motivation for this study lies in the construction of diffusion processes in spaces of interval partitions and $\mathbb{R}$-trees, which we explore in forthcoming work; see [19]. Specifically, local times correspond to branch lengths in the context of $\mathbb{R}$ trees.

This paper is structured, as follows. In Section 2, we discuss some fluctuation theory of Lévy processes and prove Proposition 4. In Section 3, we prove Proposition 5, Proposition 8, Theorem 1 and Corollary 2. In Section 4, we establish Proposition 6 and Corollary 7 using a local time approximation by jump heights, which is uniform on a sequence of refining spatial lattices in regions where local time is bounded below. Section 5 studies spectrally positive Lévy processes restricted to an interval and proves Theorem 3. Finally, Section 6 establishes Hölder constants with all moments for squared Bessel processes, excursions and bridges and related processes. This provides a class of examples to which the main results of this paper apply.

2. Preliminaries on spectrally positive stable Lévy processes. Recall the occupation density formula that characterises the local times $\left(\ell^{y}(t), y \in \mathbb{R}, t \geq 0\right)$ of $X$ :

$$
\begin{aligned}
\int_{0}^{T} f\left(X_{t}\right) d t= & \int_{-\infty}^{\infty} f(y) \ell^{y}(T) d y \\
& \text { a.s., for all } f: \mathbb{R} \rightarrow \mathbb{R} \text { bounded measurable, } T \geq 0 .
\end{aligned}
$$

The following lemma is easily obtained using the positivity parameter $1-1 /(1+$ $\alpha$ ), spatial homogeneity and scaling properties of $X$; see, for example, Bertoin [4], Sections V.1,VIII.1, for background. 
LEMMA 9. The Laplace exponent of the inverse local time $\tau^{0}$ of $X$ is $(1+$ $\alpha) \xi^{1-1 /(1+\alpha)}$.

Denote by $n, \widehat{n}$ and $\underline{\hat{n}}$ the excursion measures of $X, \widehat{X}=-X$ and $\widehat{X}-\widehat{I}=$ $S-X$ away from 0 , where $\widehat{I}_{t}=\inf \left\{\widehat{X}_{s}, s \leq t\right\}$ and $S_{t}=\sup \left\{X_{s}, s \leq t\right\}, t \geq 0$, are the running infimum and supremum of $\widehat{X}$ and $X$, respectively. We denote by $H(\omega)=A(\omega)+B(\omega)$ the size of the jump of an excursion $\omega$ across level 0 , where $A(\omega)=-\omega\left(T_{\geq 0}-\right)$ and $B(\omega)=\omega\left(T_{\geq 0}\right)$ are the undershoot and overshoot at the crossing time $T_{\geq 0}(\omega)=\inf \{t>0: \omega(t) \geq 0\}$. Similarly, $\widehat{A}(\omega)=\omega\left(T_{\leq 0}-\right)$ and $\widehat{B}(\omega)=-\omega\left(T_{\leq 0}\right)$ for $T_{\leq 0}(\omega)=\inf \{t>0: \omega(t) \leq 0\}$. We are interested in the relative undershoot $U=A / H$ jointly with $H$ under $n$, equivalently $\widehat{U}=\widehat{A} / \widehat{H}$ jointly with $\widehat{H}=\widehat{A}+\widehat{B}$ under $\widehat{n}$. Under $\underline{\hat{n}}$, the relevant crossing is the end of the excursion, at $T_{0}(\omega)=\inf \{t>0: \omega(t)=0\}$.

Proof of Proposition 4. By [34], Theorem 3, we have

$$
\begin{aligned}
\widehat{n}\left(1_{\left\{t<T_{\leq 0}\right\}} f\left(\omega\left(T_{\leq 0}-\right),-\omega\left(T_{\leq 0}\right)\right)\right) \\
\quad=\underline{\hat{n}}\left(1_{\left\{t<T_{0}\right\}} \int f(y,-z) K(\omega(t), d y, d z)\right),
\end{aligned}
$$

where $K(x, d y, d z)=(W(x)-W(x-y)) \Pi(d z-y) d y$ on $(-\infty, 0) \times(0, \infty)$, with $W(x)=(\Gamma(1+\alpha))^{-1} x^{\alpha}, x>0$, the scale function of $X$ and $\Pi(d x)=$ $(1+\alpha) \alpha(\Gamma(1-\alpha))^{-1} x^{-\alpha-2} d x$ the Lévy measure of $X$. We also denote by $u(t, x)=p_{x}(t)$ the bivariate renewal density of the decreasing ladder processes of $X$, where $p_{y}(t)$ is the density of $\sigma_{y}$ at $t$ for a $\sigma=\left(\sigma_{y}, y \geq 0\right)$ with Laplace exponent $\Phi(\xi)=\xi^{1 /(1+\alpha)}$. Specifically, we can read from [28], Sections 7.3, 8.1 and 8.2, that $\int_{0}^{\infty} e^{-\eta y} W(y) d y=1 / \eta^{1+\alpha}$ and $\int_{0}^{\infty} \int_{0}^{\infty} e^{-\xi t-\eta x} u(t, x) d x d t=$ $1 /\left(\xi^{1 /(1+\alpha)}+\eta\right)$, and these Laplace transforms are easily inverted. From [16], equation (5.2.5), we take the entrance law $\underline{\hat{n}}\left(t<T_{0}, \omega(t) \in d x\right)=c^{\prime} u(t, x) d x$, up to a constant $c^{\prime} \in(0, \infty)$. Hence,

$$
\begin{aligned}
\widehat{\underline{n}}\left(1_{\{t<} T_{0}\right\} & \left.\int f(y,-z) K(\omega(t), d y, d z)\right) \\
= & \frac{c^{\prime} \alpha(1+\alpha) \Gamma(1+\alpha)}{\Gamma(1-\alpha)} \\
& \times \int_{0}^{\infty} p_{x}(t)\left(\int_{0}^{x}\left(x^{\alpha}-(x-y)^{\alpha}\right) \int_{-\infty}^{0} f(y,-z)(y-z)^{-\alpha-2} d z d y\right. \\
& \left.+\int_{x}^{\infty} x^{\alpha} \int_{-\infty}^{0} f(y,-z)(y-z)^{-\alpha-2} d z d y\right) d x .
\end{aligned}
$$

To compute this, and to let $t \downarrow 0$, we apply Fubini's theorem and first consider the 
$x$-integral over the $(x-y)^{\alpha}$-term:

$$
\begin{aligned}
\int_{y}^{\infty} & p_{x}(t)(x-y)^{\alpha} d x \\
& =\int_{0}^{\infty} p_{r+y}(t) r^{\alpha} d r \\
& =\int_{0}^{\infty} p_{1}\left((r+y)^{-\alpha-1} t\right)(r+y)^{-\alpha-1} r^{\alpha} d r \\
& =t^{-\alpha /(1+\alpha)} \frac{1}{1+\alpha} \int_{0}^{t y^{-1-\alpha}} p_{1}(s) s^{-1 /(1+\alpha)}\left(t^{1 /(1+\alpha} s^{-1 /(1+\alpha)}-y\right)^{\alpha} d s \\
& \leq \frac{1}{1+\alpha} \int_{0}^{\infty} 1_{\left\{s \leq t y^{-1-\alpha\}}\right.} p_{1}(s) s^{-1} d s,
\end{aligned}
$$

by the dominated convergence theorem, as the integral without the indicator equals $\Gamma(1 / \alpha) / \alpha$ (see, e.g., [40], equation (0.40)). The remaining terms yield, using similar substitutions,

$$
\begin{aligned}
& \frac{c^{\prime} \alpha(1+\alpha) \Gamma(1+\alpha)}{\Gamma(1-\alpha)} \\
& \times \frac{1}{1+\alpha} \int_{0}^{\infty} p_{1}(s) s^{-1} d s \int_{0}^{\infty} \int_{-\infty}^{0} f(y,-z)(y-z)^{-\alpha-2} d z d y \\
& \quad=\frac{c^{\prime} \Gamma(1+\alpha) \Gamma(1 / \alpha)}{\Gamma(1-\alpha)} \int_{0}^{\infty} \int_{0}^{\infty} f(y, z)(y+z)^{-\alpha-2} d z d y .
\end{aligned}
$$

We conclude that under the excursion measure $n$ of $X=-\widehat{X}$, undershoot $A$ and overshoot $B$ satisfy

$$
n(A \in d y, B \in d z)=\frac{c^{\prime} \Gamma(1+\alpha) \Gamma(1 / \alpha)}{\Gamma(1-\alpha)}(y+z)^{-\alpha-2} d z d y
$$

and hence $H=A+B$ and $U=A / H$ satisfy

$$
n(H \in d x, U \in d r)=\frac{c^{\prime} \Gamma(1+\alpha) \Gamma(1 / \alpha)}{\Gamma(1-\alpha)} x^{-\alpha-1} d x d r .
$$

In particular, $U=A /(A+B)$ is uniformly distributed under $n(\cdot \mid H=x)$ for a.e. $x>0$ and indeed for all $x>0$ by the scaling property. The remaining results are classical. The independence claim is a consequence of the Markov property under $n$ (see, e.g., [4], Section IV.4) as is the conditional distribution of the post-jump process under $n(\cdot \mid A=y, B=z)$. The claim about the pre-jump process follows by time-reversal [20]. Since the two first hitting times are downward level passage times, their sum has Laplace transform given by

$$
n\left(\exp \left(-\xi T_{0}\right) \mid A=y, B=z\right)=\exp (-(y+z) \Phi(\xi)) .
$$


On the other hand, by Lemma 9, the Laplace exponent of $\tau^{0}$ is $(1+\alpha) \xi^{1-1 /(1+\alpha)}$. Therefore,

$$
\begin{aligned}
n\left(1-\exp \left(-\xi T_{0}\right)\right) & =(1+\alpha) \xi^{1-1 /(1+\alpha)} \\
& =\int_{0}^{\infty}\left(1-\exp \left(-x \xi^{1 /(1+\alpha)}\right)\right) \frac{(1+\alpha) \alpha}{\Gamma(1-\alpha)} x^{-\alpha-1} d x,
\end{aligned}
$$

so we require

$$
\frac{c^{\prime} \Gamma(1+\alpha) \Gamma(1 / \alpha)}{\Gamma(1-\alpha)} x^{-\alpha-1}=\frac{(1+\alpha) \alpha}{\Gamma(1-\alpha)} x^{-\alpha-1},
$$

that is,

$$
c^{\prime}=\frac{(1+\alpha) \alpha}{\Gamma(1+\alpha) \Gamma(1 / \alpha)} .
$$

3. Uniform approximation of local times. We will use notation $\widehat{m}_{h}(y, r)=$ $m_{h}\left(y, \tau^{y}(r)\right)-m_{h}\left(y, \tau^{y}(0)\right)$. The purpose of this section is to prove Theorem 1 . The argument is inspired by Khoshnevisan [27], who studies rates of convergence. We only need weaker results. Weaker results have also been obtained by different methods for different classes of processes, for example, [2, 3, 12, 35], but Khoshnevisan's methods seem most adaptable to our setting. Khoshnevisan uses excursion theory to study intrinsic approximations of Brownian local times based on excursion lengths. Excursion lengths while intrinsic to the level set are not intrinsic to the population sizes $Z_{t}\left(y-X_{t-}\right), 0 \leq t \leq T$, at level $y$, while our approximations in Theorem 1 have this latter property.

\subsection{Proofs of Proposition 5 and Proposition 8.}

ProOF OF PROPOSITION 5. First, note that $\kappa_{q}(x, \cdot)$ is the distribution of $\left(x^{q} Z(s / x), 0 \leq s \leq x\right)$ with Hölder constant

$$
\sup _{0 \leq r<s \leq x} \frac{\left|x^{q} Z(s / x)-x^{q} Z(r / x)\right|}{|s-r|^{\gamma}}=x^{q-\gamma} D_{\gamma} .
$$

For all $t \in[0, T]$ with $\Delta X_{t}>0$, denote the associated Hölder constant by

$$
d_{t}=\sup _{0 \leq r<s \leq \Delta X_{t}} \frac{\left|Z_{t}(s)-Z_{t}(r)\right|}{|s-r|^{\gamma}},
$$

and set $d_{t}=0$ if $\Delta X_{t}=0$. Now clearly, for all $p>0$,

$$
\begin{aligned}
& \left(\sup \left\{d_{t}: 0 \leq t \leq T\right\}\right)^{p} \\
& \quad \leq \max \left\{\left(\sup \left\{d_{t}^{p}: 0 \leq t \leq T, \Delta X_{t}>1\right\}\right)^{p}, \sum_{0 \leq t \leq T: \Delta X_{t} \leq 1} d_{t}^{p}\right\}
\end{aligned}
$$


and by the compensation formula for Poisson point processes,

$$
\mathbb{E}\left(\sum_{0 \leq t \leq T: \Delta X_{t} \leq 1} d_{t}^{p}\right)=T \frac{(1+\alpha) \alpha}{\Gamma(1-\alpha)} \mathbb{E}\left(\left(D_{\gamma}\right)^{p}\right) \int_{0}^{1} x^{p(q-\gamma)-\alpha-2} d x<\infty,
$$

provided that $p>(1+\alpha) /(q-\gamma)$. This completes the proof, since there are at most finitely many jumps of size exceeding 1 in $[0, T]$, as their rate is finite in the Poisson point process.

Using the same argument, we also obtain the following result.

LEMMA 10. Let $\theta \in(0, \gamma]$ and let $\widehat{d}_{t}$ denote the $\theta$-Hölder constant of $Z_{t}$

$$
\widehat{d}_{t}:=\sup _{0 \leq r<s \leq \Delta X_{t}} \frac{\left|Z_{t}(s)-Z_{t}(r)\right|}{(s-r)^{\theta}} .
$$

For every $\varepsilon>0$, there is some nonrandom $C_{\varepsilon}>0$ such that for every $z \in(0,1]$,

$$
\mathbb{E}\left(\sup _{0 \leq t \leq T: 0<\Delta X_{t} \leq z} \widehat{d}_{t}\right)<C_{\varepsilon} z^{q-\theta-\varepsilon}
$$

Proof. Fix $\theta \in(0, \gamma]$ and let $p>(1+\alpha) /(q-\theta)$. When also $p \geq 1$, we apply Jensen's inequality and argue as in the proof of Proposition 5, via the scaling invariance of $\kappa_{q}$, to find that

$$
\begin{aligned}
\mathbb{E}\left(\sup _{0 \leq t \leq T: \Delta X_{t} \leq z} \widehat{d}_{t}\right) & \leq\left(\mathbb{E}\left(\sum_{0 \leq t \leq T: \Delta X_{t} \leq z} \widehat{d}_{t}^{p}\right)\right)^{1 / p} \\
& =\left(\mathbb{E}\left(\sum_{0 \leq t \leq T: \Delta X_{t} \leq z}\left(\Delta X_{t}\right)^{(q-\theta) p}\right)\right)^{1 / p}\left(\mathbb{E}\left(D_{\theta}^{p}\right)\right)^{1 / p} \\
& =\left(\mathbb{E}\left(D_{\theta}^{p}\right)\right)^{1 / p}\left(\int_{0}^{z} x^{(q-\theta) p} \frac{(1+\alpha) \alpha T}{\Gamma(1-\alpha)} x^{-\alpha-2} d x\right)^{1 / p} \\
& =\left(\mathbb{E}\left(D_{\theta}^{p}\right)\right)^{1 / p} C_{p}^{\prime} z^{q-\theta-(1+\alpha) / p}
\end{aligned}
$$

where $C_{p}^{\prime}$ is a finite deterministic term that depends on $p$. Since $D_{\theta}$ has moments of all orders, the RHS above is finite for all $p>(1+\alpha) /(q-\theta), p \geq 1$. To obtain (5), we take $p>(1+\alpha) / \varepsilon$.

ProOf of PROpOSITION 8. First, consider $y=0$. Recall notation $n$ for the Itô excursion measure of $X$ away from $0, H$ for the size of the jump across 0 and $U$ for the relative undershoot of that jump across 0 . We applied the marking kernel $\kappa_{q}$ to the Poisson point process of all jumps of $X$, and this induces a $\kappa_{q}$-mark $Z_{T_{\geq 0}}$ of the jump $\left(T_{\geq 0}, H\right)$ under $n$. We write $n_{+}(d \omega, d f)=\kappa_{q}(H(\omega), d f) n(d \omega)$ for the 
intensity measure of the Itô excursion process with marked jump across 0 . Recall the joint density of $(H, U)$ under $n$ from Proposition 4.

By (1), the jumps of $t \mapsto Z_{[0, t]}(0)$ will be of the form $J_{t}=Z_{t}\left(-X_{t-}\right)$, where $-X_{t-}$ is the undershoot of any jump of $X$ at time $t$ across level 0 . Since $X$ is spectrally positive, there is at most one such jump per excursion. A standard mapping argument shows that those jumps form a Poisson point process in the inverse local time parametrisation of the Itô excursion process, with intensity measure

$$
\begin{aligned}
& n_{+}(\{(\omega, f): f(H(\omega) U(\omega))>h\}) \\
& \quad=\int_{0}^{\infty} \int_{0}^{1} \mathbb{P}\left(x^{q} Z(u)>h\right) \frac{(1+\alpha) \alpha}{\Gamma(1-\alpha)} x^{-\alpha-1} d x d u \\
& \quad=\int_{0}^{\infty} \mathbb{P}(Z(U)>z) \frac{(1+\alpha) \alpha}{\Gamma(1-\alpha) q} h^{-(1+\alpha) / q+1 / q} z^{(1+\alpha) / q-1 / q-1} d z \\
& \quad=\frac{1+\alpha}{\Gamma(1-\alpha)} h^{-\alpha / q} \mathbb{E}\left((Z(U))^{\alpha / q}\right)=c h^{-\alpha / q},
\end{aligned}
$$

identifying $c$ as given in the statement of Theorem 1 . We note that this establishes part (ii) of Proposition 8. For (i), we obtain that the $J_{t}$ are summable for $q>\alpha$, as we recognise the tail of the stable Lévy measure of index $\alpha / q$, with Laplace exponent:

$$
\Theta(\xi)=\int_{(0, \infty)}\left(1-e^{-\xi h}\right) n_{+}(f(H(\omega) U(\omega)) \in d h)=c \Gamma(1-\alpha / q) \xi^{\alpha / q} .
$$

The generalisation from $y=0$ to general $y \in \mathbb{R}$ follows by spatial homogeneity of $X$ and by the strong Markov property of $X$ at $T_{y}=\inf \left\{t>0: X_{t}=y\right\}$, since $T_{y} \geq T_{\geq y}$ a.s.

3.2. Auxiliary results for the proof of Theorem 1. To prove Theorem 1, we will need a moderate deviations result for Poisson processes, which we deduce from standard large deviations results as can be found, for example, in Dembo and Zeitouni [15].

LEMMA 11. Let $\left(N_{t}, t \geq 0\right)$ be a standard Poisson process with $N_{t} \sim \operatorname{Poi}(t)$. Then for all $z>\delta>0$, there is $t_{0} \geq 1$ such that, for all $t \geq t_{0}$,

$$
\mathbb{P}\left(\left|N_{t}-t\right| \geq \sqrt{t \log (t)} \sqrt{2 z}\right) \leq t^{-z+\delta} .
$$

ProOF. We apply [15], Theorem 3.7.1, to independent centred Poisson variables $Y_{i}=N_{i}-N_{i-1}-1, i \geq 1$ and $a_{n}=(2 z \log (n))^{-1}, n \geq 1$. Since $a_{n} \rightarrow 0$ and $n a_{n} \rightarrow \infty$ as $n \rightarrow \infty$, we find that for any $\varepsilon>0$, there is $n_{0} \geq 1$ such that for all $n \geq n_{0}$ :

$$
\mathbb{P}\left(\sum_{i=1}^{n} Y_{i} \geq \sqrt{\frac{n}{a_{n}}}\right) \leq \exp \left(-\frac{1}{2 a_{n}}\right)=n^{-z}
$$


To pass from integer $n \geq 1$ to real $t \geq 2$, we note that

$$
\begin{aligned}
& \mathbb{P}\left(N_{t}-t \geq \sqrt{t \log (t)} \sqrt{2 z}\right) \\
& \quad \leq \mathbb{P}\left(N_{\lceil t\rceil}-\lceil t\rceil \geq-1+\sqrt{(\lceil t\rceil-1) \log (\lceil t\rceil-1)} \sqrt{2 z}\right)
\end{aligned}
$$

is of the same form, with $\sqrt{n / a_{n}}$ replaced by

$$
\sqrt{n / b_{n}}=-1+\sqrt{(n-1) \log (n-1)} \sqrt{2 z},
$$

and another application of [15], Theorem 3.7.1, yields, for $n=\lceil t\rceil$,

$$
\mathbb{P}\left(N_{t}-t \geq \sqrt{t \log (t)} \sqrt{2 z}\right) \leq \exp \left(-\frac{1}{2 b_{n}}\right) \leq\lceil t\rceil^{-z+\delta / 2} \leq t^{-z+\delta / 2},
$$

for $t \geq n_{0}$, for a possibly increased $n_{0}$. A similar argument deals with $\mathbb{P}\left(t-N_{t} \geq\right.$ $\sqrt{t \log (t)} \sqrt{2 z}$ ), and together, possibly increasing $n_{0}$ again, we obtain the stated result.

By Proposition 8, we have $\widehat{m}_{h}(y, r) \sim \operatorname{Poi}\left(r c h^{-\alpha / q}\right)$. Moreover, $t \mapsto N_{t}(y, r)=$ $\widehat{m}_{\left(r c t^{-1}\right)^{q / \alpha}}(y, r), t>0$ is a standard Poisson process, by independence properties of the Poisson point process of jumps of $s \mapsto Z_{\left[0, \tau^{y}(s)\right]}(y)$. We will apply the previous lemma to a variation of this Poisson process. Following Khoshnevisan [27], Lemmas 5.1-5.3, we consider an independent $\lambda \sim \operatorname{Exp}(1)$.

Lemma 12. Let $T_{y}=\inf \left\{t \geq 0: X_{t}=y\right\}, y \in \mathbb{R}$. Then for all $y>0$, we have

$$
\mathbb{P}\left(T_{-y}<\lambda\right)=\exp (-y)
$$

and

$$
\mathbb{P}\left(T_{y}<\lambda\right)=\frac{1+\alpha}{\pi} \int_{0}^{\infty} \frac{\sin (\pi \alpha) s^{1+\alpha}}{s^{2(1+\alpha)}+2 s^{1+\alpha} \cos (\pi \alpha)+1} e^{-y s} d s
$$

Proof. Let $y>0$. Since $X$ is spectrally positive, we have $T_{-y}=\inf \{t \geq$ $\left.0: X_{t} \leq-y\right)$, and it is well known that $\mathbb{P}\left(T_{-y}<\lambda\right)=\mathbb{E}\left(e^{-T_{-y}}\right)=\exp (-y)$; see, for example, Bertoin [4], Chapter VII, for first passage problems of spectrally negative Lévy processes such as $-X$. On the other hand, Simon [46] showed that, $T_{1} \sim R T_{-1}$, where $R$ is independent of $T_{-1}$ with probability density function

$$
f_{R}(t)=\frac{\sin (\pi \alpha) t^{1 /(1+\alpha)}}{\pi\left(t^{2}+2 t \cos (\pi \alpha)+1\right)} 1_{\{t \geq 0\}} .
$$

By scaling, $T_{y} \sim y^{1+\alpha} T_{1} \sim y^{1+\alpha} R T_{-1} \sim R T_{-y}$. With this, we obtain

$$
\begin{aligned}
\mathbb{P}\left(T_{y}<\lambda\right) & =\mathbb{P}\left(T_{-y} R<\lambda\right) \\
& =\frac{1}{\pi} \int_{0}^{\infty} \frac{\sin (\pi \alpha) t^{1 /(1+\alpha)}}{t^{2}+2 t \cos (\pi \alpha)+1} \mathbb{P}\left(t T_{-y}<\lambda\right) d t
\end{aligned}
$$




$$
\begin{aligned}
& =\frac{1}{\pi} \int_{0}^{\infty} \frac{\sin (\pi \alpha) t^{1 /(1+\alpha)}}{t^{2}+2 t \cos (\pi \alpha)+1} \mathbb{P}\left(T_{-y t} 1^{1 /(1+\alpha)}<\lambda\right) d t \\
& =\frac{1}{\pi} \int_{0}^{\infty} \frac{\sin (\pi \alpha) t^{1 /(1+\alpha)}}{t^{2}+2 t \cos (\pi \alpha)+1} e^{-y t^{1 /(1+\alpha)}} d t \\
& =\frac{1+\alpha}{\pi} \int_{0}^{\infty} \frac{\sin (\pi \alpha) s^{1+\alpha}}{s^{2(1+\alpha)}+2 s^{1+\alpha} \cos (\pi \alpha)+1} e^{-y s} d s .
\end{aligned}
$$

LEMMA 13. For all $y \in \mathbb{R}$, we have

$$
\mathbb{P}\left(\ell^{y}(\lambda)>r\right)=\mathbb{P}\left(T_{y}<\lambda\right) \exp (-(1+\alpha) r) .
$$

ProOF. By the strong Markov property and spatial homogeneity of $X$, we have

$$
\mathbb{P}\left(\ell^{y}(\lambda)>a\right)=\mathbb{P}\left(T_{y}<\lambda\right) \mathbb{P}\left(\ell^{0}(\lambda)>a\right) .
$$

To calculate $\mathbb{P}\left(\ell^{0}(\lambda)>a\right)$, note that by the strong Markov property of $X$ at inverse local times, $\ell^{0}(\lambda)$ is exponentially distributed, and by Lemma 9 ,

$$
\begin{aligned}
\mathbb{E}\left(\ell^{0}(\lambda)\right) & =\int_{0}^{\infty} \mathbb{E}\left(\ell^{0}(t)\right) e^{-t} d t=\int_{0}^{\infty} \int_{0}^{\infty} \mathbb{P}\left(\ell^{0}(t)>s\right) d s e^{-t} d t \\
& =\int_{0}^{\infty} \int_{0}^{\infty} \mathbb{P}\left(\tau^{0}(s)<t\right) e^{-t} d t d s \\
& =\int_{0}^{\infty} \mathbb{E}\left(e^{-\tau^{0}(s)}\right) d s=\frac{1}{1+\alpha},
\end{aligned}
$$

that is, $\ell^{0}(\lambda)$ has distribution $\operatorname{Exp}(1+\alpha)$, as claimed.

Recall notation $\widehat{m}_{h}(y, r)=m_{h}\left(y, \tau^{y}(r)\right)-m_{h}\left(y, \tau^{y}(0)\right)$ for the Poisson counting process of level- $y$ excursions of $X$ with path-mark exceeding $h$ at the crossing level, parametrised by level- $y$ local time $r \geq 0$. Recall from Proposition 8(ii) that its rate is $c h^{-\alpha / q}$. Note that for each $y \in \mathbb{R}$, the inverse local time $\tau^{y}(0)$ equals the first hitting time $T_{y}$ of level $y$ a.s., and that $m_{h}\left(y, \tau^{y}(0)\right)=1$ if the single jump of $X$ across level $y$ before $T_{y}$ has a path-mark exceeding $h$, while $m_{h}\left(y, \tau^{y}(0)\right)=0$ otherwise.

We will study $m_{h}(y, \lambda)$ by first investigating $\widehat{m}_{h}\left(y, \ell^{y}(\lambda)-\right)$, that is, the excursion count stopped just before the excursion straddling the independent exponential time $\lambda$. On the event $\left\{\lambda \leq T_{y}\right\}$, we have $\ell^{y}(\lambda)=0$ and $\widehat{m}_{h}\left(y, \ell^{y}(\lambda)-\right)=0$. On the event $\left\{\lambda>T_{y}\right\}$, we consider $\lambda$ as a further mark of the excursion of $X$ straddling $\lambda$, and we can represent the stopped excursion count as a thinned Poisson process $r \mapsto \tilde{m}_{h}(y, r)$ stopped at an independent time $\ell^{y}(\lambda)$, which by Lemma 13 
has distribution $\operatorname{Exp}(1+\alpha)$. The thinned Poisson process has rate $c h^{-\alpha / q}-a_{h}$ for some $a_{h} \in[0,1+\alpha]$. Specifically,

$$
a_{h}=\int_{0}^{\infty} \int_{0}^{1} \mathbb{P}\left(x^{q} Z(u)>h\right) \frac{\alpha(1+\alpha}{\Gamma(1-\alpha)} x^{-\alpha-1}\left(1-e^{-x}\right) d u d x,
$$

where $1-e^{-x}=\mathbb{P}(\lambda<x)$ is the thinning probability and $Z \sim \kappa_{q}(1, \cdot)$. See, for example, [44], Section VI.49, for details on the thinning of excursion processes.

Finally, note that $m_{h}(y, \lambda)-m_{h}\left(y, \tau^{y}(0)\right)-\widehat{m}_{h}\left(y, \ell^{0}(\lambda)-\right)=1$ if the excursion straddling $\lambda$ has crossed before $\lambda$ with a path-mark exceeding $h$ at the crossing level, while $m_{h}(y, \lambda)-m_{h}\left(y, \tau^{y}(0)\right)-\widehat{m}_{h}\left(y, \ell^{0}(\lambda)-\right)=0$ otherwise. In summary, we have for each $y \in \mathbb{R}$,

$$
\widehat{m}_{h}\left(y, \ell^{y}(\lambda)-\right) \leq m_{h}(y, \lambda) \leq \widehat{m}_{h}\left(y, \ell^{y}(\lambda)-\right)+2 \quad \text { a.s. }
$$

LEMMA 14. For all $r_{0}>0, \theta>0, \varepsilon>0$, there is $h_{0}>0$ such that for all $h \leq h_{0}, y \in \mathbb{R}$,

$$
\begin{aligned}
& \mathbb{P}\left(\left|\frac{h^{\alpha / q}}{c} \widehat{m}_{h}\left(y, \ell^{y}(\lambda)-\right)-\ell^{y}(\lambda)\right|\right. \\
& \left.\quad \geq \sqrt{\frac{2(\theta+\varepsilon)(1+2 \varepsilon)}{c}} h^{\alpha / 2 q} \sqrt{\log \left(\frac{1}{h}\right)} \sqrt{\ell^{y}(\lambda)}, \ell^{y}(\lambda) \geq r_{0}\right) \leq h^{\theta} .
\end{aligned}
$$

PROOF. Recall that $q>\alpha$. Let $r_{0}>0$ and $\theta>0$ and, without loss of generality, $\varepsilon>0$ so small that we have $\varepsilon q(\theta+\varepsilon)\left(2+7 \varepsilon^{2}+4 \varepsilon^{4}\right)<q-\alpha$. Then we apply the strong Markov property of $X$ at $T_{y}$ and the spatial homogeneity of $X$ to find that

$$
\begin{aligned}
p_{h}:= & \mathbb{P}\left(\left|\frac{h^{\alpha / q}}{c} \widehat{m}_{h}\left(y, \ell^{y}(\lambda)-\right)-\ell^{y}(\lambda)\right|\right. \\
& \left.\geq \sqrt{\frac{2(\theta+\varepsilon)(1+2 \varepsilon)}{c}} h^{\alpha / 2 q} \sqrt{\log \left(\frac{1}{h}\right)} \sqrt{\ell^{y}(\lambda)}, \ell^{y}(\lambda) \geq r_{0}\right) \\
= & \mathbb{P}\left(T_{y}<\lambda\right) \mathbb{P}\left(\left|\frac{h^{\alpha / q}}{c} \widehat{m}_{h}\left(0, \ell^{0}(\lambda)-\right)-\ell^{0}(\lambda)\right|\right. \\
& \left.\geq \sqrt{\frac{2(\theta+\varepsilon)(1+2 \varepsilon)}{c}} h^{\alpha / 2 q} \sqrt{\log \left(\frac{1}{h}\right)} \sqrt{\ell^{0}(\lambda)}, \ell^{0}(\lambda) \geq r_{0}\right) \\
\leq & \int_{r_{0}}^{\infty} \mathbb{P}\left(\left|\frac{h^{\alpha / q}}{c} \tilde{m}_{h}(0, r)-r\right|\right. \\
& \left.\geq \sqrt{\frac{2(\theta+\varepsilon)(1+2 \varepsilon)}{c}} h^{\alpha / 2 q} \sqrt{\log \left(\frac{1}{h}\right)} \sqrt{r}\right) \mathbb{P}\left(\ell^{0}(\lambda) \in d r\right),
\end{aligned}
$$


where $\widetilde{m}_{h}(0, \cdot)$ is the thinning of $\widehat{m}_{h}(0, \cdot)$ independent of $\ell^{0}(\lambda)$ as discussed above the statement of the lemma. As a function of $h>0$, for fixed $r$, we can write $\tilde{m}_{h}(0, r)=N\left(r\left(c h^{-\alpha / q}-a_{h}\right)\right)$ in terms of a unit rate Poisson process $N$, to which Lemma 11 applies. Due to the thinning, we need to recentre to get a deviation probability from the mean in the integrand above:

$$
\begin{aligned}
& \mathbb{P}\left(\left|N\left(r\left(c h^{-\alpha / q}-a_{h}\right)\right)-r c h^{-\alpha / q}\right|\right. \\
& \left.\geq \sqrt{r c h^{-\alpha / q} \log \left(\frac{1}{h}\right)} \sqrt{2(\theta+\varepsilon)(1+2 \varepsilon)}\right) \\
& \quad \leq \mathbb{P}\left(\left|N\left(r\left(c h^{-\alpha / q}-a_{h}\right)\right)-r\left(c h^{-\alpha / q}-a_{h}\right)\right|\right. \\
& \left.\geq \sqrt{r c h^{-\alpha / q} \log \left(\frac{1}{h}\right)} \sqrt{2(\theta+\varepsilon)(1+2 \varepsilon)}-r a_{h}\right) .
\end{aligned}
$$

The deviation threshold is not quite of the form to which Lemma 11 applies either, but after a few more steps, we will apply Lemma 11 for $z:=(\theta+\varepsilon)\left(1-2 \varepsilon^{2}-\right.$ $\left.7 \varepsilon^{4}-4 \varepsilon^{6}\right) q / \alpha$ and $\delta:=z-\theta q / \alpha-\varepsilon=\varepsilon\left(q / \alpha-1-\varepsilon(\theta+\varepsilon)\left(2+7 \varepsilon^{2}+4 \varepsilon^{4}\right) q / \alpha\right)$, which satisfy $z>\delta>0$ by the restriction we have put onto $\varepsilon$. Let $t_{0} \geq 1$ be obtained from Lemma 11 and set

$$
\begin{aligned}
h_{0}:= & \min \left\{\frac{1}{e},\left(\frac{\sqrt{c}}{1+\alpha} \varepsilon^{2} \sqrt{2(\theta+\varepsilon)(1+2 \varepsilon)}\right)^{2(1-2 \varepsilon) q / \alpha(1-3 \varepsilon)},\right. \\
& \left.c^{-(1-2 \varepsilon) q / \alpha \varepsilon},\left(\frac{c r_{0}}{(1+\alpha) r_{0}+t_{0}}\right)^{q / \alpha}\right\} .
\end{aligned}
$$

Now let $r \leq h^{-\varepsilon \alpha / q(1-2 \varepsilon)}$. First, we turn the $r a_{h}$ term on the right-hand side into $\varepsilon^{2}$ times the square-root term: for all $h \leq h_{0}$,

$$
a_{h} \leq 1+\alpha, \quad \sqrt{r} \leq h^{-\varepsilon \alpha / 2 q(1-2 \varepsilon)} \quad \text { and } \quad 1 \leq \log (1 / h),
$$

using the first bound on $h_{0}$ and find that our claim is equivalent to the second bound on $h_{0}$. Then we check that by the third bound on $h_{0}$

$$
c \leq h^{-\varepsilon \alpha / q(1-2 \varepsilon)} \quad \text { and hence } \log \left(\frac{1}{h}\right) \geq \frac{q}{\alpha}(1-2 \varepsilon) \log \left(r c h^{-\alpha / q}\right),
$$

for all $h \leq h_{0}$. Finally, we estimate $r c h^{-\alpha / q} \geq r c h^{-\alpha / q}-r a_{h}$ and note that $r c h^{-\alpha / q}-r a_{h} \geq t_{0} \geq 1$ for all $h \leq h_{0}$ is equivalent to the fourth bound on $h_{0}$. Hence, Lemma 11 applies and yields an upper bound for our integrand of

$$
\begin{aligned}
& \mathbb{P}\left(\left|N\left(r\left(c h^{-\alpha / q}-a_{h}\right)\right)-r\left(c h^{-\alpha / q}-a_{h}\right)\right|\right. \\
& \quad \geq \sqrt{r\left(c h^{-\alpha / q}-a_{h}\right) \log \left(r\left(c h^{-\alpha / q}-a_{h}\right)\right)} \sqrt{\left.\frac{2(\theta+\varepsilon)\left(1-4 \varepsilon^{2}\right)\left(1+\varepsilon^{2}\right)^{2} q}{\alpha}\right)}
\end{aligned}
$$




$$
\begin{aligned}
& \leq\left(r\left(c h^{-\alpha / q}-a_{h}\right)\right)^{-\theta q / \alpha-\varepsilon} \\
& \leq\left(c-(1+\alpha) h_{0}^{\alpha / q}\right)^{-\theta q / \alpha-\varepsilon} h^{\theta+\varepsilon \alpha / q} r^{-\theta q / \alpha-\varepsilon},
\end{aligned}
$$

so that $p_{h}$ is bounded above by

$$
\begin{aligned}
(c- & \left.(1+\alpha) h_{0}^{\alpha / q}\right)^{-\theta q / \alpha-\varepsilon} h^{\theta+\varepsilon \alpha / q} \int_{r_{0}}^{h^{-\varepsilon \alpha / q(1-2 \varepsilon)}} r^{-\theta q / \alpha-\varepsilon} \mathbb{P}\left(\ell^{0}(\lambda) \in d r\right) \\
+ & \mathbb{P}\left(\ell^{0}(\lambda)>h^{-\varepsilon \alpha / q(1-2 \varepsilon)}\right) \\
\leq & \left(r_{0} c-r_{0}(1+\alpha) h_{0}^{\alpha / q}\right)^{-\theta q / \alpha-\varepsilon} h^{\theta+\varepsilon \alpha / q} \\
& \quad+\exp \left(-(1+\alpha) h^{-\varepsilon \alpha / q(1-2 \varepsilon)}\right) \\
\leq & h^{\theta}
\end{aligned}
$$

for all $h \leq h_{0}$, possibly by decreasing $h_{0}$ further to accommodate the last inequality.

Now fix $r_{0}>0, \varepsilon>0$ and $\theta>0$. Following Khoshnevisan [27], we define for any $\mu>0, R>0, h>0$ and $y \in \mathbb{R}$ the lattice $K(\mu, R)=(-R, R) \cap \mu \mathbb{Z}$ and the events $G(y, h)$ given by

$$
\begin{aligned}
& \left\{\left|\frac{h^{\alpha / q}}{c} \widehat{m}_{h}\left(y, \ell^{y}(\lambda)-\right)-\ell^{y}(\lambda)\right|\right. \\
& \left.\quad \geq \sqrt{\frac{2(\theta+\varepsilon)(1+2 \varepsilon)}{c}} h^{\alpha / 2 q} \sqrt{\log (1 / h)} \sqrt{\ell^{y}(\lambda)}, \ell^{y}(\lambda) \geq r_{0}\right\} .
\end{aligned}
$$

COROLlaRy 15. For any $w>0$ and $r>0$, let $\rho(k)=k^{-w}$ and $\mu(k)=$ $(\rho(k))^{r}=k^{-w r}$. Then

$$
\begin{aligned}
& \limsup _{k \rightarrow \infty} \sup _{y \in K(\mu(k), R)} \frac{\left|\frac{(\rho(k))^{\alpha / q}}{c} m_{\rho(k)}(y, \lambda)-\ell^{y}(\lambda)\right|}{(\rho(k))^{\alpha / 2 q} \sqrt{\log (1 / \rho(k))}} 1_{\left\{\ell^{y}(\lambda) \geq r_{0}\right\}} \\
& \leq \sqrt{\frac{2(r+1 / w)}{c} \sqrt{\ell^{*}(\lambda)}} \quad \text { a.s., }
\end{aligned}
$$

where $\ell^{*}(\lambda)=\sup _{y \in \mathbb{R}} \ell^{y}(\lambda)$.

PROOF. First, note by (6) and the triangular inequality that it is equivalent to prove the statement with $m_{\rho(k)}(y, \lambda)$ replaced by $\widehat{m}_{\rho(k)}\left(y, \ell^{y}(\lambda)-\right)$, since $2(\rho(k))^{\alpha / q} / c(\rho(k))^{\alpha / 2 q} \sqrt{\log (1 / \rho(k))} \rightarrow 0$. Then this proof becomes a simple application of the Borel-Cantelli lemma: for all $\theta>r+1 / w$,

$$
\sum_{k \geq 1} \mathbb{P}\left(\bigcup_{y \in K(\mu(k), R)} G(y, \rho(k))\right) \leq 2 R \sum_{k \geq 1} \frac{(\rho(k))^{\theta}}{\mu(k)}<\infty
$$


implies that a.s.

$$
\begin{aligned}
& \limsup _{k \rightarrow \infty} \sup _{y \in K(\mu(k), R)} \frac{\left|\frac{(\rho(k))^{\alpha / q}}{c} m_{\rho(k)}(y, \lambda)-\ell^{y}(\lambda)\right|}{(\rho(k))^{\alpha / 2 q} \sqrt{\log (1 / \rho(k))}} 1_{\left\{\ell^{y}(\lambda) \geq r_{0}\right\}} \\
& \quad \leq \sqrt{\frac{2(\theta+\varepsilon)(1+2 \varepsilon)}{c}} \sqrt{\ell^{*}(\lambda)}
\end{aligned}
$$

and considering countable sequences $\varepsilon_{n} \downarrow 0$ and $\theta_{n} \downarrow r+1 / w$, we obtain the bound given.

We seek to strengthen the corollary by replacing the supremum over $K(\mu(k), R)$ by a supremum over $[-R, R]$.

Proposition 16. For all $R>0$ and $r_{0}>0$, we have

$$
\begin{gathered}
\limsup _{h \downarrow 0} \sup _{y \in[-R, R]} \frac{\left|\frac{h^{\alpha / q}}{c} m_{h}(y, \lambda)-\ell^{y}(\lambda)\right|}{h^{\alpha / 2 q} \sqrt{\log (1 / h)}} 1_{\left\{\ell^{y}(\lambda) \geq 2 r_{0}\right\}} \\
\leq \sqrt{\frac{2 q+\alpha(\gamma+1)}{q \gamma c}} \sqrt{\ell^{*}(\lambda)} \quad \text { a.s. }
\end{gathered}
$$

PROOF. Let $D_{[0, \lambda]}$ be as in Proposition 5, for $T=\lambda$. We will work on the event of probability 1 , where $D_{[0, \lambda]}<\infty, y \mapsto \ell^{y}(\lambda)$ satisfies Boylan's [9] modulus of continuity $\left|\ell^{y}(\lambda)-\ell^{x}(\lambda)\right| \leq K_{\lambda}|\log | y-x||(|y-x|)^{\alpha / 2}$ and where the bound of the preceding corollary holds. There is $k_{0} \geq 2$ for which the following two estimates hold. First, we can guarantee for all $k \geq k_{0}$ that

$$
D_{[0, \lambda]}(\mu(k-1))^{\gamma} \leq \rho(k-1)-\rho(k)
$$

and

$$
D_{[0, \lambda]}(\mu(k+1))^{\gamma} \leq \rho(k)-\rho(k+1),
$$

provided that $r>(1+1 / w) / \gamma$. Second, we can guarantee that $\left|\ell^{y}(\lambda)-\ell^{z}(\lambda)\right| \leq$ $r_{0}$ for all $y, z \in[-R, R]$ with $|y-z| \leq \mu\left(k_{0}\right)$. For $y \in[-R, R]$ and $k \geq 1$, let $g_{k}(y) \in K(\mu(k), R)$ with $\left|g_{k}(y)-y\right| \leq \mu(k)$. Then for all $k \geq k_{0}$, we have on the chosen event of probability 1 ,

$$
\begin{aligned}
\ell^{y}(\lambda) & -\frac{(\rho(k))^{\alpha / q}}{c} m_{\rho(k)}(y, \lambda) \\
\leq & \left(\frac{\rho(k)}{\rho(k-1)}\right)^{\alpha / q}\left(\ell^{g_{k-1}(y)}(\lambda)-\frac{(\rho(k-1))^{\alpha / q}}{c} m_{\rho(k-1)}\left(g_{k-1}(y), \lambda\right)\right) \\
& +\left|\ell^{y}(\lambda)-\ell^{g_{k-1}(y)}(\lambda)\right|+\left(1-\left(\frac{\rho(k)}{\rho(k-1)}\right)^{\alpha / q}\right) \ell^{*}(\lambda) .
\end{aligned}
$$


We divide both sides by $(\rho(k))^{\alpha / 2 q} \sqrt{\log (1 / \rho(k))}$ and take suprema $y \in[-R, R]$. Adding indicators $1_{\left\{\ell^{y}(\lambda) \geq 2 r_{0}\right\}}$ on the LHS, we may add indicators $1\left\{\ell^{g_{k-1}(y)}(\lambda) \geq\right.$ $\left.r_{0}\right\}$ on the first term of the RHS. With these denominators, suprema and indicators, the first term of the RHS has limsup as stated in the preceding corollary, for all $k \geq k_{0}$, since $\rho(k) / \rho(k-1) \rightarrow 1$, The other two terms vanish in the limit, since $r>1 / \gamma>1 / q$, provided that $w \leq 2 q / \alpha$, which implies $(1-(\rho(k) / \rho(k-$ $\left.1))^{\alpha / q}\right) /\left(\rho(k)^{\alpha / 2 q} \log (1 / \rho(k))\right) \rightarrow 0$. Similarly, the suprema $y \in[-R, R]$ of the following bounds have bounded limsup when adding the analogous indicators and denominators:

$$
\begin{aligned}
& \frac{(\rho(k))^{\alpha / q}}{c} m_{\rho(k)}(y, \lambda)-\ell^{y}(\lambda) \\
& \quad \leq\left(\frac{\rho(k)}{\rho(k+1)}\right)^{\alpha / q}\left(\frac{(\rho(k+1))^{\alpha / q}}{c} m_{\rho(k+1)}\left(g_{k+1}(y), \lambda\right)-\ell^{g_{k+1}(y)}(\lambda)\right) \\
& \quad+\left|\ell^{y}(\lambda)-\ell^{g_{k+1}(y)}(\lambda)\right|+\left(\left(\frac{\rho(k)}{\rho(k+1)}\right)^{\alpha / q}-1\right) \ell^{*}(\lambda) .
\end{aligned}
$$

To strengthen the limit along $h=\rho(k) \rightarrow 0$ to $h \downarrow 0$, write $\bar{h}=\min (\{\rho(k), k \geq$ $1\} \cap[h, \infty))$ and $\underline{h}=\max (\{\rho(k), k \geq 1\} \cap(0, h])$. Then we can bound above

$$
\begin{aligned}
& \frac{h^{\alpha / q}}{c} m_{h}(y, \lambda)-\ell^{y}(\lambda) \\
& \quad \leq\left(\frac{\bar{h}}{\underline{h}}\right)^{\alpha / q}\left(\frac{\underline{h}^{\alpha / q}}{c} m_{\underline{h}}(y, \lambda)-\ell^{y}(\lambda)\right)+\left(\left(\frac{\bar{h}}{\underline{h}}\right)^{\alpha / q}-1\right) \ell^{*}(\lambda),
\end{aligned}
$$

similarly below. A straightforward argument completes the proof since $w \leq 2 q / \alpha$ so that $\bar{h} / \underline{h} \rightarrow 1$ can be strengthened to $(\bar{h} / \underline{h})^{\alpha / q}-1=o\left(h^{\alpha / 2 q} \log (1 / h)\right)$ as $h \downarrow 0$, and with $w=2 q / \alpha$ and $r_{n} \downarrow(1+\alpha / 2 q) / \gamma$, we establish the bound $(2 q+\alpha(\gamma+$ 1)) $/ q \gamma c$ as claimed.

Note that Proposition 16 is weaker than Theorem 1 in the sense that it is not uniform in $t$ and subject to a bound $2 r_{0}$ on the local times. On the other hand, Proposition 16 is stronger than Theorem 1 in the sense that it establishes rates of convergence of at least $h^{\alpha / 2 q} \sqrt{\log (1 / h)}$. In the proof of Theorem 1, we will apply Proposition 16, but we could not avoid sacrificing the rate of convergence to establish uniformity in $t$ and to remove the restricting bound $2 r_{0}$.

\subsection{Proofs of Theorem 1 and Corollary 2.}

Proof of TheOREM 1. For the proof of the theorem, first note that Proposition 16 gives a result at a single $\operatorname{Exp}(1)$ distributed random time. Now fix $R>0$ 
and consider for all $t>0$ and $r_{0}>0$ the events

$$
\begin{aligned}
A\left(t, r_{0}, R\right)= & \left\{\limsup _{h \downarrow 0} \sup _{y \in[-R, R]} \frac{\left|\frac{h^{\alpha / q}}{c} m_{h}(y, t)-\ell^{y}(t)\right|}{h^{\alpha / 2 q} \sqrt{\log (1 / h)}} 1_{\left\{\ell^{y}(t) \geq 2 r_{0}\right\}}\right. \\
& \left.\leq \sqrt{\frac{2 q+\alpha(\gamma+1)}{q \gamma c}} \sqrt{\ell^{*}(t)}\right\} .
\end{aligned}
$$

Then we have shown that $1=\mathbb{P}\left(A\left(\lambda, r_{0}, R\right)\right)=\int_{0}^{\infty} \mathbb{P}\left(A\left(t, r_{0}, R\right)\right) e^{-t} d t$, hence $\mathbb{P}\left(A\left(t, r_{0}, R\right)\right)=1$ for all $t \geq 0$ except possibly on a Lebesgue null set. Let us give a scaling argument to prove that this null set must be empty. Recall the scaling property of the stable process $X$ that $X_{a}(t)=a^{-1 /(1+\alpha)} X(a t), t \geq 0$, is identical in distribution to $X$, for all $a>0$. This entails $\ell_{a}^{y}(t)=a^{-\alpha /(1+\alpha)} \ell^{a^{1 /(1+\alpha)} y}(a t)$ and $m_{h}^{a}(y, t)=m_{a^{q /(1+\alpha)} h}\left(a^{1 /(1+\alpha)} y, a t\right)$ and $A_{a}\left(t, r_{0}, R\right)=A\left(a t, a^{\alpha /(1+\alpha)} r_{0}\right.$, $\left.a^{1 /(1+\alpha)} R\right)$, using obvious notation. The arguments together yield that for Lebesgue-a.e. $s \geq 0$ and all $a \in[1 / 2,2]$

$$
\begin{aligned}
1 & =\mathbb{P}\left(A_{a}\left(s, r_{0} / 2^{\alpha /(1+\alpha)}, 2^{1 /(1+\alpha)} R\right)\right) \\
& =\mathbb{P}\left(A\left(a s,(a / 2)^{\alpha /(1+\alpha)} r_{0},(2 a)^{1 /(1+\alpha)} R\right)\right) \leq \mathbb{P}\left(A\left(a s, r_{0}, R\right)\right),
\end{aligned}
$$

and this entails that $\mathbb{P}\left(A\left(t, r_{0}, R\right)\right)=1$ for all $t>0$, and, trivially, also for $t=0$.

Now fix $T>t>0$ and let $\varepsilon>0$ with $t, \varepsilon \in \mathbb{Q}$. With $r_{0}=\varepsilon / 2$, we can a.s. find $h_{0}>0$ such that for all $h<h_{0}$

$$
\begin{aligned}
& \sup _{y \in[-R, R]}\left(\ell^{y}(t)-\frac{h^{\alpha / q}}{c} m_{h}(y, t)\right) \\
& \quad \leq \varepsilon+\left(\varepsilon+\sqrt{\frac{2 q+\alpha(\gamma+1)}{q \gamma c}} \sqrt{\ell^{*}(t)}\right) h^{\alpha / 2 q} \sqrt{\log \left(\frac{1}{h}\right)} .
\end{aligned}
$$

Taking $\lim \sup _{h \downarrow 0}$ and $\varepsilon \downarrow 0$ along a sequence $\varepsilon_{n} \downarrow 0$, we see that almost surely

$$
\limsup \sup _{h \downarrow 0}\left(\ell_{y \in[-R, R]}^{y}(t)-\frac{h^{\alpha / q}}{c} m_{h}(y, t)\right) \leq 0 .
$$

But on the event $\left\{-R \leq \inf _{0 \leq t \leq T} X_{t} \leq \sup _{0 \leq t \leq T} X_{t} \leq R\right\}$, this supremum is actually $\sup _{y \in \mathbb{R}}$. Also, (7) holds for all $t \in \mathbb{Q} \cap(0, T]$ a.s. By continuity of $t \mapsto \ell^{y}(t)$ (uniformly in $y$ ), and the monotonicity of $t \mapsto m_{h}(y, t)$, this holds for all $t \in[0, \infty)$ a.s. Furthermore, for all $\varepsilon>0$, there is $\delta>0$ so that for all $y \in \mathbb{R}$ and all $s<t$ in $[0, T],|s-t|<\delta$ implies $\ell^{y}(t)-\ell^{y}(s)<\varepsilon$. So,

$$
\limsup _{h \downarrow 0} \sup _{y \in[-R, R]} \sup _{s \in \delta \mathbb{Z} \cap[0, T]}\left(\ell^{y}(s)-\frac{h^{\alpha / q}}{c} m_{h}(y, s)\right) \leq 0
$$

already entails

$$
\limsup _{h \downarrow 0} \sup _{y \in[-R, R]} \sup _{t \in[0, T]}\left(\ell^{y}(t)-\frac{h^{\alpha / q}}{c} m_{h}(y, t)\right) \leq \varepsilon .
$$


Taking a rational sequence $\varepsilon_{n} \downarrow 0$, this limsup must vanish a.s. This argument worked for fixed $R>0$ on the event $\left\{-R \leq \inf _{0 \leq t \leq T} X_{t} \leq \sup _{0 \leq t \leq T} X_{t} \leq R\right\}$. Now taking the union of these events over a rational sequence $R_{n} \uparrow \infty$, we conclude that we have the "upper bound"

$$
\limsup _{h \downarrow 0} \sup _{y \in \mathbb{R}} \sup _{t \in[0, T]}\left(\ell^{y}(t)-\frac{h^{\alpha / q}}{c} m_{h}(y, t)\right) \leq 0 \quad \text { a.s. }
$$

For the "lower bound", we have similarly for all $R>0, t \in \mathbb{Q} \cap[0, \infty), r_{0}>0$,

$$
\limsup _{h \downarrow 0} \sup _{y \in[-R, R]: \ell y}(t) \geq 2 r_{0}\left(\frac{h^{\alpha / q}}{c} m_{h}(y, t)-\ell^{y}(t)\right) \leq 0 \quad \text { a.s. }
$$

Now let $\varepsilon>0$ and choose $r_{0}=\varepsilon / 4$. Let $S>0$. On the event

$$
\left\{-R \leq \inf _{0 \leq t \leq T} X_{t} \leq \sup _{0 \leq t \leq T} X_{t} \leq R\right\} \cap\left\{\ell^{y}(T+S) \geq \varepsilon / 2 \text { for all } y \in[-R, R]\right\},
$$

we find $h_{0}$ such that for all $h<h_{0}$

$$
\sup _{y \in \mathbb{R}} \sup _{t \in \delta \mathbb{Z} \cap[0, T+S]: \ell^{y}(t) \geq 2 r_{0}}\left(\frac{h^{\alpha / q}}{c} m_{h}(y, t)-\ell^{y}(t)\right) \leq \varepsilon \quad \text { a.s., }
$$

where we choose $\delta>0$ so small that $|t-s|<\delta$ in $[0, T+S]$ implies that $\mid \ell^{y}(t)-$ $\ell^{y}(s) \mid<\varepsilon$. We define $\tau^{y}(\varepsilon)=\inf \left\{t \geq 0: \ell^{y}(t) \geq \varepsilon\right\}$. Then we conclude that

$$
\limsup \sup _{h \downarrow 0} \sup _{y \in \mathbb{R}}\left(\frac{h^{\alpha / q}}{c} m_{h}(y, t)-\ell^{y}(t)\right) \leq 3 \varepsilon
$$

since for any $y \in \mathbb{R}$ and $t \in[0, T]$ with $\ell^{y}(t)<2 r_{0}$ and $s$ the next lattice point in $\delta \mathbb{Z}$ after $\tau^{y}(\varepsilon)$, we can estimate for $h \leq h_{0}$

$$
\begin{aligned}
\frac{h^{\alpha / q}}{c} m_{h}(y, t)-\ell^{y}(t) & \leq \frac{h^{\alpha / q}}{c} m_{h}\left(y, \tau^{y}(\varepsilon / 3)\right) \\
& \leq \frac{h^{\alpha / q}}{c} m_{h}\left(y, \tau^{y}(\varepsilon)\right)-\ell^{y}(s)+2 \varepsilon \leq 3 \varepsilon \quad \text { a.s. }
\end{aligned}
$$

For sequences $\varepsilon_{n} \downarrow 0$ and $R_{n} \uparrow \infty$, this completes the proof, as for the "upper bound".

Proof OF COROLlary 2. This follows straight from Theorem 1. Just note that by symmetry of $z$

$$
\begin{aligned}
\frac{1}{c^{\circ}} & =c=(1+\alpha)(\Gamma(1-\alpha))^{-1} 2 \int_{0}^{1 / 2} s^{\alpha} d s \\
& =2 \frac{1}{\Gamma(1-\alpha)} \frac{1}{2^{1+\alpha}}=\frac{1}{2^{\alpha} \Gamma(1-\alpha)} .
\end{aligned}
$$


4. Uniform Hölder continuity of the paths in $\kappa_{q}$-marked stable processes. To prove Proposition 6 and Corollary 7, we will use a local time approximation based directly on jump height, which we present first.

4.1. Local time approximations based on jump heights. Consider the count $m_{h}^{\prime}(y, T)=\#\left\{0 \leq t \leq T: X_{t-}<y<X_{t}, \Delta X_{t}>h\right\}$. As in Proposition 8, it follows from Proposition 4 that $\widehat{m}_{h}^{\prime}(y, r)=m_{h}^{\prime}\left(y, \tau^{y}(r)\right)-m_{h}^{\prime}\left(y, \tau^{y}(0)\right)$ is a Poisson process with rate $c^{\prime} h^{-\alpha}$ where $c^{\prime}=(1+\alpha) \alpha / \Gamma(1-\alpha)$, and the Poisson process arguments in the proof of Lemma 14, as well as the Borel-Cantelli argument for Corollary 15 apply again to give for an independent $\lambda \sim \operatorname{Exp}(1)$.

LEMMA 17. For all $r_{0}>0, \theta>0, \varepsilon>0$, there is $h_{0}>0$ such that for all $h \leq h_{0}, y \in \mathbb{R}$,

$$
\begin{aligned}
& \mathbb{P}\left(\left|\frac{h^{\alpha}}{c^{\prime}} \widehat{m}_{h}^{\prime}\left(y, \ell^{y}(\lambda)-\right)-\ell^{y}(\lambda)\right|\right. \\
& \left.\quad \geq \sqrt{\frac{2(\theta+\varepsilon)(1+2 \varepsilon)}{c^{\prime}}} h^{\alpha / 2} \sqrt{\log \left(\frac{1}{h}\right)} \sqrt{\ell^{y}(\lambda)}, \ell^{y}(\lambda) \geq r_{0}\right) \leq h^{\theta} .
\end{aligned}
$$

COROLlary 18. For any $w>0$ and $r>0$, let $\rho(k)=k^{-w}$ and $\mu(k)=$ $(\rho(k))^{r}=k^{-w r}$. Then

$$
\begin{aligned}
& \limsup _{k \rightarrow \infty} \sup _{y \in K(\mu(k), R)} \frac{\left|\frac{\rho(k)^{\alpha}}{c^{\prime}} m_{\rho(k)}^{\prime}(y, \lambda)-\ell^{y}(\lambda)\right|}{(\rho(k))^{\alpha / 2} \sqrt{\log (1 / \rho(k))}} 1_{\left\{\ell^{y}(\lambda) \geq r_{0}\right\}} \\
& \leq \sqrt{2(r+1 / w)} c^{\prime} \sqrt{\ell^{*}(\lambda)} \quad \text { a.s. }
\end{aligned}
$$

where $\ell^{*}(\lambda)=\sup _{y \in \mathbb{R}} \ell^{y}(\lambda)$.

The proof of Proposition 16, however, exploits uniform Hölder bounds on $Z_{t}$, $t \in[0, T]$, to show that for all $k$ large enough, we have

$$
m_{\rho(k-1)}\left(g_{k-1}(y), \lambda\right) \leq m_{\rho(k)}(y, \lambda) \leq m_{\rho(k+1)}\left(g_{k+1}(y), \lambda\right),
$$

where $g_{k}(y)$ is the nearest lattice point to $y$ in $K(\mu(k), R)$. This argument would need substantial change since a substitute for the Hölder bounds would need to be found to control the number of jumps greater than $\rho(k)$ that cross level $y$. It is, however, straightforward to find much weaker upper bounds, such as the following, which will be enough to prove Proposition 6.

Proposition 19. We have almost surely for all $T \geq 0$

$$
\limsup _{h \downarrow 0} \sup _{y \in \mathbb{R}} h^{\alpha} m_{h}^{\prime}(y, T)<\infty \text {. }
$$


PROOF. Let $\varepsilon>0$. We first claim $\limsup _{h \downarrow 0} \sup _{y \in \mathbb{R}} h^{\alpha} m_{h}^{\prime}(y, \lambda) 1_{\left\{\ell^{y}(\lambda) \geq 2 r_{0}\right\}} \leq$ $2 c^{\prime} \ell^{*}(\lambda)$ a.s. By Corollary 18 , there is $k_{1} \geq 1$ such that for all $k \geq k_{1}$ and all $y \in$ $K(\mu(k), R+1)$ with $\ell^{y}(\lambda) \geq r_{0}$

$$
\begin{aligned}
& \rho(k)^{\alpha} \widehat{m}_{\rho(k)}^{\prime}(y, \lambda) \\
& \quad \leq c^{\prime} \ell^{y}(\lambda)+c^{\prime}(\rho(k))^{\alpha / 2} \sqrt{\log (1 / \rho(k))}\left(\sqrt{2(r+1 / w)} c^{\prime} \sqrt{\ell^{*}(\lambda)}+\varepsilon\right) \\
& \quad \leq c^{\prime} \ell^{*}(\lambda)+c^{\prime}(\rho(k))^{\alpha / 2} \sqrt{\log (1 / \rho(k))}\left(\sqrt{2(r+1 / w)} c^{\prime} \sqrt{\ell^{*}(\lambda)}+\varepsilon\right) .
\end{aligned}
$$

Let $r>1$. Then we have $\mu(k)<\rho(k)$. For $y \in[-R, R]$, let $g_{k}^{+}(y)=\inf K(\mu(k)$, $R+1) \cap[y, \infty)$ and $g_{k}^{-}(y)=\sup K(\mu(k), R+1) \cap(-\infty, y]$ be the lattice points of $K(\mu(k), R+1)$ nearest to $y$. As in the proof of Proposition 16, we find $k_{0} \geq k_{1}$ such that $\left|\ell^{y}(\lambda)-\ell^{z}(\lambda)\right| \leq r_{0}$ for all $y, z \in[-R, R]$ with $|y-z| \leq \mu\left(k_{0}\right)$. Then for all $y \in[-R, R]$ with $\ell^{y}(\lambda) \geq 2 r_{0}$, we have $\ell^{g_{k}^{+}(y)}(\lambda) \geq r_{0}$ and $\ell^{g_{k}^{-}(y)}(\lambda) \geq r_{0}$.

For all $0 \leq t \leq T$ with $X_{t-}<y<X_{t}$ and $\Delta X_{t}>\rho(k)>\mu(k)$, we must have $X_{t-}<g_{k}^{+}(y)<X_{t}$ or $X_{t-}<g_{k}^{-}(y)<X_{t}$. Hence,

$$
m_{\rho(k)}^{\prime}(y, \lambda) \leq m_{\rho(k)}^{\prime}\left(g_{k}^{+}(y), \lambda\right)+m_{\rho(k)}^{\prime}\left(g_{k}^{-}(y), \lambda\right),
$$

so that for all $y \in[-R, R]$ with $\ell^{y}(\lambda) \geq 2 r_{0}$, we have

$$
\begin{aligned}
& \rho(k)^{\alpha} m_{\rho(k)}^{\prime}(y, \lambda) \\
& \quad \leq 2 c^{\prime} \ell^{*}(\lambda)+2 c^{\prime}(\rho(k))^{\alpha / 2} \sqrt{\log (1 / \rho(k))}\left(\sqrt{2(r+1 / w)} c^{\prime} \sqrt{\ell^{*}(\lambda)}+\varepsilon\right) .
\end{aligned}
$$

To get from $h=\rho(k) \rightarrow 0$ to $h \downarrow 0$, recall notation $\bar{h}=\min (\{\rho(k), k \geq 1\} \cap$ $[h, \infty))$ and $\underline{h}=\max (\{\rho(k), k \geq 1\} \cap(0, h])$. Then we can bound above

$$
h^{\alpha} m_{h}^{\prime}(y, \lambda) \leq\left(\frac{\bar{h}}{\underline{h}}\right)^{\alpha} \underline{h}^{\alpha} m_{\underline{h}}^{\prime}(y, \lambda)
$$

and hence conclude by letting $h \downarrow 0$ to see the upper bound independent of $y$ tend to $2 c^{\prime} \ell^{*}(\lambda)$ to find

(9) $\quad \limsup _{h \downarrow 0} \sup _{y \in[-R, R]} h^{\alpha} m_{h}^{\prime}(y, \lambda) 1_{\left\{\ell^{y}(\lambda) \geq 2 r_{0}\right\}} \leq 2 c^{\prime} \ell^{*}(\lambda) \quad$ almost surely.

Since $\mathcal{R}=\left(X_{t}, 0 \leq t \leq \lambda\right)$ is bounded almost surely, the claim hence holds on the events $\{\mathcal{R} \subset[-R, R]\}$ whose union over $R \in \mathbb{N}$ has probability 1 , so it remains to remove the indicator.

Now let $T \geq 0$ and define the post- $T$ process $\tilde{X}_{t}=X_{T+t}-X_{T}$, with local times $\tilde{\ell}^{y}(t)=\ell^{y-X_{T}}(T+t)-\ell^{y-X_{T}}(T), y \in \mathbb{R}, t \geq 0$. Note that $\tilde{X}$ is independent of $\left(X_{t}, 0 \leq t \leq T\right)$ with the same distribution as $X$. For $R \in \mathbb{N}$, let

$$
E_{R}=\left\{\tilde{\ell}^{y}(\lambda)>2 r_{0} \text { for all } y \in[-R, R]\right\} .
$$


If we can prove that each of these events $E_{R}$ has positive probability, we obtain via (9) that

$$
\begin{aligned}
\underset{h \downarrow 0}{\limsup } & \sup _{y \in\left[X_{T}-R, X_{T}+R\right]} h^{\alpha} m_{h}^{\prime}(y, T) \\
& \leq \limsup _{h \downarrow 0} \sup _{y \in\left[X_{T}-R, X_{T}+R\right]} h^{\alpha} m_{h}^{\prime}(y, T+\lambda)<\infty
\end{aligned}
$$

almost surely on $E_{R}$. But $E_{R}$ is independent of $\left(X_{t}, 0 \leq t \leq T\right)$; thus, the term on the LHS is a.s. finite for every $R$. Let $M:=S_{T}-I_{T}=\sup \left\{X_{S}, s \leq t\right\}-\inf \left\{X_{s}, s \leq\right.$ $t\}$. This is a.s. finite. In the event $\{M<R\}$, the inequality above implies (8). Since this event happens for some $R \in \mathbb{N}$, (8) holds almost surely. It remains to show that $E_{R}$ has positive probability, which we do restate and prove in the following lemma.

LEMMA 20. Let $Z \sim \operatorname{Exp(1).~Then~for~all~} \varepsilon>0$ and all $k>0$, the event $E_{R}$ of the proof of Proposition 19 has positive probability. In particular, with probability $1, \ell^{y}(t) \rightarrow \infty$ as $t \rightarrow \infty$ for all $y \in \mathbb{R}$, uniformly on compact $y$-intervals.

Proof. Since $\tau^{0}$ is a stable subordinator, $\ell^{0}(\lambda)>0$ a.s., hence there is $\varepsilon>0$ such that $\ell^{0}(\lambda)>2 \varepsilon$ with positive probability. But then by continuity of $y \mapsto$ $\ell^{y}(\lambda)$, there is $R>0$ such that with positive probability, we have $\ell^{y}(\lambda)>\varepsilon$ for all $y \in[-R, R]$.

Now fix $R>0$ and consider $\tau^{0}(j), j \geq 1$. Then the random variables $\min \left\{\ell^{y}\left(\tau^{0}(j)\right)-\ell^{y}\left(\tau^{0}(j-1)\right), y \in[-R, R]\right\}, j \geq 1$, are independent and identically distributed, nonnegative and positive with positive probability. By the strong law of large numbers, their series is infinite a.s., and so $\ell^{y}(\infty)=\infty$ for all $y \in[-R, R]$, a.s. By scaling, this holds for all $R>0$, and by choosing a sequence $R_{i} \rightarrow \infty, i \rightarrow \infty$, this extends to all $y \in \mathbb{R}$, as required.

\subsection{Proofs of Proposition 6 and Corollary 7.}

Proof of Proposition 6. Let $J_{i}$ be the time of the $i$ th largest jump of $\left(X_{t}, 0 \leq t \leq T\right)$ and recall that $Z_{t}$ is the excursion marking the jump of $X$ at time $t$. We build our partition sequentially and refer to the resulting sequences $\left(J_{j}^{k}, j \geq 1\right)$ as "piles", $k \geq 1$ [2].

Piling procedure. Place $J_{1}$ at the bottom of the first pile, $J_{1}^{1}:=J_{1}$. Now suppose that the first $i$ jump times are arranged into $k_{i}$ piles of heights $j_{1}, \ldots, j_{k_{i}}$, with $\sum_{k \leq k_{i}} j_{k}=i$.

- If for each $k \leq k_{i}$ there exists $j \leq j_{k}$ such that

$$
\left[X\left(J_{i+1}-\right), X\left(J_{i+1}\right)\right] \cap\left[X\left(J_{j}^{k}-\right), X\left(J_{j}^{k}\right)\right] \neq \varnothing
$$

then we place it $J_{i+1}$ into a new pile by setting $J_{1}^{k_{i}+1}:=J_{i+1}$. 
- Otherwise, we place $J_{i+1}$ atop the pile of least index $k$ for which

$$
\left[X\left(J_{j+1}-\right), X\left(J_{j+1}\right)\right] \cap\left[X\left(J_{j}^{k}-\right), X\left(J_{j}^{k}\right)\right]=\varnothing
$$

for every $j \leq j_{k}$, that is, $J_{j_{k}+1}^{k}:=J_{i+1}$, where

$$
\begin{aligned}
k= & \min \left\{m \leq k_{i}:\left[X\left(J_{i+1}-\right), X\left(J_{i+1}\right)\right]\right. \\
& \left.\cap\left[X\left(J_{j}^{m}-\right), X\left(J_{j}^{m}\right)\right]=\varnothing \text { for all } j \in\left[j_{m}\right]\right\} .
\end{aligned}
$$

We denote the $\theta$-Hölder constants by

$$
D_{j}^{k}:=\sup _{0 \leq a<b \leq \Delta X\left(J_{j}^{k}\right)} \frac{\left|Z_{J_{j}^{k}}(b)-Z_{J_{j}^{k}}(a)\right|}{(b-a)^{\theta}}, \quad j \geq 1
$$

and

$$
D_{[0, T]}^{k}:=\sup _{j \geq 1} D_{j}^{k}, \quad \text { for all } k \geq 1 .
$$

By definition of our piling procedure, $J_{1}^{k}$ is the time of the largest jump in the $k$ th pile, for each $k \geq 1$. Consider the start and end levels $a_{k}=X\left(J_{1}^{k}-\right)$ and $b_{k}=$ $X\left(J_{1}^{k}\right)$ of the jump at time $J_{1}^{k}$. Again, by definition of the procedure, for each $m<k$ there is some jump time $J_{j}^{m}$ that is the time of a larger jump than $J_{1}^{k}$ and such that the jump intervals intersect

$$
\left[X\left(J_{1}^{k}-\right), X\left(J_{1}^{k}\right)\right] \cap\left[X\left(J_{j}^{m}-\right), X\left(J_{j}^{m}\right)\right] \neq \varnothing .
$$

Since $\Delta X\left(J_{1}^{k}\right)<\Delta X\left(J_{j}^{m}\right)$, for each such $J_{j}^{m}$, the jump interval contains one of the endpoints of the jump at time $J_{k}^{1}$ :

$$
\left\{X\left(J_{1}^{k}-\right), X\left(J_{1}^{k}\right)\right\} \cap\left[X\left(J_{j}^{m}-\right), X\left(J_{j}^{m}\right)\right] \neq \varnothing .
$$

By the pigeonhole principle, and the fact that almost surely there is no level at which more than one jump starts or ends, there is some $y_{k} \in\left(X\left(J_{1}^{k}-\right), X\left(J_{1}^{k}\right)\right)$ such that at least $\left\lfloor\frac{k}{2}\right\rfloor$ jumps larger than $\Delta X_{J_{1}^{k}}$ jump across level $y_{k}$. That is,

$$
\#\left\{i \geq 1: \Delta X\left(J_{i}\right)>\Delta X\left(J_{1}^{k}\right) \text { and } y_{k} \in\left[X\left(J_{i}-\right), X\left(J_{i}\right)\right]\right\} \geq\left\lfloor\frac{k}{2}\right\rfloor .
$$

Proposition 19 implies that there is almost surely some $C \in(0, \infty)$ such that for every $y \in \mathbb{R}$ and every $k \geq 1$, the $k$ th largest jump across level $y$ has size at most $C k^{-1 / \alpha}$. Since $\Delta X\left(J_{1}^{k}\right)$ is at most the $\left\lfloor\frac{k}{2}\right\rfloor$ th largest jump across level $y_{k}$, we see that $\Delta X\left(J_{1}^{k}\right) \leq C\left\lfloor\frac{k}{2}\right\rfloor^{-1 / \alpha} \leq C^{\prime} k^{-1 / \alpha}$. Thus $\Delta X\left(J_{j}^{k}\right) \leq C^{\prime} k^{-1 / \alpha}$ for all $j \geq 1$.

Take $\varepsilon>0$. Then, from the above, there is a.s. some finite $K$ such that for $k>K$, the jump size $\Delta X\left(J_{1}^{k}\right)$ is at most $k^{\varepsilon-1 / \alpha}$. Let

$$
\begin{aligned}
D^{*} & :=\sup _{k \geq 1, j \geq 1} D_{j}^{k} \quad \text { and } \\
D^{\prime} & :=\sum_{m \geq 1} \sup \left\{D_{j}^{k}: \Delta X\left(J_{1}^{k}\right)<m^{\varepsilon-1 / \alpha}, k \geq 1, j \geq 1\right\},
\end{aligned}
$$


so that

$$
\sum_{n \geq 1} D_{[0, T]}^{n} \leq K D^{*}+D^{\prime} .
$$

From Proposition 5, $D^{*}$ is a.s. finite, and $K$ is a.s. finite as well, so it suffices to show that $D^{\prime}$ is a.s. finite. We appeal to (5), applied with $z=m^{\varepsilon-1 / \alpha} \leq 1$ if $\varepsilon<1 / \alpha$, to find that

$$
\mathbb{E}\left(D^{\prime}\right) \leq \sum_{m \geq 1} C_{\varepsilon} m^{\left(\varepsilon-\frac{1}{\alpha}\right)(q-\theta-\varepsilon)} .
$$

Since $\theta \leq \gamma$ and $q>\gamma+\alpha$, this series converges for all sufficiently small $\varepsilon$.

Proof OF COROLlary 7. For this proof, abbreviate $Z_{t}^{y}:=Z_{t}\left(y-X_{t-}\right)$ and $Z_{t}^{x}:=Z_{t}\left(x-X_{t-}\right)$. Then

$$
\begin{aligned}
\left|Z_{[0, T]}(y)-Z_{[0, T]}(x)\right| & =\left|\sum_{0 \leq t \leq T}\left(Z_{t}^{y}-Z_{t}^{x}\right)\right| \leq \sum_{0 \leq t \leq T}\left|Z_{t}^{y}-Z_{t}^{x}\right| \\
& \leq \sum_{0 \leq t \leq T: Z_{t}^{y} \neq 0}\left|Z_{t}^{y}-Z_{t}^{x}\right|+\sum_{0 \leq t \leq T: Z_{t}^{x} \neq 0}\left|Z_{t}^{y}-Z_{t}^{x}\right| \\
& \leq\left(2 \sum_{n \geq 1} D_{n}\right)|y-x|^{\gamma}
\end{aligned}
$$

by Proposition 6.

5. Uniform Hölder continuity of local times. Boylan [9] established that the family of local times of $(1+\alpha)$-stable Lévy processes admits a version that is Hölder continuous of order $v \in(0, \alpha / 2)$ in the spatial direction, uniformly in space and time on any compact space-time rectangles. Barlow [1] gave the exact modulus of continuity. While Barlow's results are optimal in rate, we are interested in a (random) Hölder constant with all moments and which applies uniformly on space-time rectangles that consist of a fixed compact spatial interval and relevant random time intervals, whose random lengths have infinite mean. To achieve this without getting into the technicalities of Barlow's argument, we sacrifice a slowly varying function in the exact modulus of continuity and restrict the stable process to a spatial interval.

5.1. Spectrally one-sided Lévy processes restricted to an interval. Consider at first any zero mean spectrally negative Lévy process $\widehat{X}$ with unbounded variation and zero Gaussian coefficient, with notation to facilitate passage to the spectrally positive process $X=-\widehat{X}$ later. For the process $\widehat{X}, 0$ is regular for itself and for $(-\infty, 0)$, by [4], Corollary VII.5. By [4], Theorems IV.4 and IV.10, $\widehat{X}$ admits a 
continuous local time at 0 , and hence at any level, with an associated excursion process, whose intensity measure we denote by $\widehat{n}$.

By [4], Proposition 1, the same holds for the reflected process $\widehat{Y}=\widehat{X}-\widehat{I}$, where $\widehat{I_{t}}=\inf _{0 \leq s \leq t} \widehat{X}_{s}, t \geq 0$; as in Section 2, we denote the excursion measure by $\underline{\hat{n}}$. The measures $\widehat{n}$ and $\underline{\hat{n}}$ are measures on the Skorohod space $\mathbb{D}([0, \infty), \mathbb{R})$, supported by the subspace of paths $\omega=(\omega(s))_{s \geq 0} \in \mathbb{D}([0, \infty), \mathbb{R})$ for which $\omega(s) \neq 0$ if and only if $s \in\left(0, T_{0}(\omega)\right)$, where $T_{0}(\omega)=\inf \{t>0: \omega(t)=0\} \in(0, \infty]$ is called the lifetime of the excursion $\omega$. Since $\widehat{X}$ is spectrally negative, $\widehat{n}$-a.e. excursion $\omega$ has at most one jump across zero, whose time we denote by $T_{\leq 0}(\omega)=\inf \{t>$ $0: \omega(t) \leq 0\}$. By [34], Lemma 2, there is exactly one jump across zero $\widehat{n}$-a.e., under our assumption of a zero Gaussian coefficient. By [34], Theorem 3,

$$
\widehat{\underline{n}}=\widehat{n}\left(\left(\omega(s) 1_{\left\{s<T_{\leq 0}\right\}}\right)_{s \geq 0} \in \cdot\right) .
$$

In particular, if we define the time change

$$
\tau^{+}(r)=\inf \left\{t \geq 0: R^{+}(t)>r\right\} \quad \text { where } R^{+}(t)=\int_{0}^{t} 1_{\left\{\widehat{X}_{s} \geq 0\right\}} d s,
$$

so that $\widehat{X}_{r}^{+}=\widehat{X}_{\tau^{+}(r)}$ has all negative parts of excursions removed, then $\widehat{X}^{+}$has the same distribution as $\widehat{Y}$. The analogous operation that removes all negative parts of excursions from the spectrally positive process $X=-\widehat{X}$ also yields a strong Markov process $X^{+}$, but this process does not have the same distribution as $Y=$ $X-I$, where $I_{t}=\inf _{0 \leq s \leq t} X_{s}$, because excursions of $Y$ start continuously (none of the countably many jumps strike during the Lebesgue-null set of times when $Y=$ 0 , by independence properties of Poisson point processes), while excursions of $X^{+}$ away from 0 start with a jump, with intensity $\chi=n\left(\omega\left(T_{\geq 0}\right) \in \cdot\right)=\widehat{n}\left(-\omega\left(T_{\leq 0}\right) \in\right.$ $\cdot)$, where $n=\widehat{n}(-\omega \in \cdot)$ is the excursion measure of $X$ at 0 and $T_{\geq 0}(\omega)=\inf \{t>$ $0: \omega(t) \geq 0\}$. By the strong Markov property under $n$ at $T_{\geq 0}$, the post- $T_{\geq 0}$ process under $n$ is the post- $T_{\geq 0}$ process of the spectrally positive Lévy process absorbed at 0 ; cf. [34], Theorem 3, and cf. also [29], Section 3, where the authors introduce stable Lévy processes that have the negative parts of their excursions away from 0 removed. By Vigon's équations amicales (see [16], Theorem 16), $\chi$ is absolutely continuous with

$$
\chi(d x)=\bar{\Pi}(x) d x=\Pi((x, \infty)) d x,
$$

where $\Pi$ is the Lévy measure of $X$ so that

$$
\psi(\eta)=\log \left(\mathbb{E}\left(\exp \left(-\eta X_{1}\right)\right)\right)=\int_{(0, \infty)}\left(e^{-\eta x}-1+\eta x\right) \Pi(d x) .
$$

Finally, we define the spectrally positive Lévy process restricted to an interval 
$[0, b]$ as $X_{r}^{b}=X_{\tau^{[0, b]}(r)}$, where

$$
\tau^{[0, b]}(r)=\inf \left\{t \geq 0: R^{[0, b]}(t)>r\right\} \quad \text { and } \quad R^{[0, b]}(t)=\int_{0}^{t} 1_{\left\{0 \leq X_{s} \leq b\right\}} d s .
$$

Note that this process is different from (actually simpler than) Pistorius' doubly reflected process of [38], since here the boundary behaviour at 0 is not reflection, and is also different from Lambert's [30] process confined in a finite interval [conditioned not to exit $(0, b)]$. The discussion of these three cases is connected to the discussion in [10] and [29], Remark 3.3, which distinguish three types of exit at a boundary (A) continuously, (B) by a jump or (C) not at all (while preserving self-similarity of the process). In our context (two boundaries, self-similarity being meaningless on an interval, but re-entry being allowed), Lambert studies $(\mathrm{C}, \mathrm{C})$ exits from $(0, b)$, no entry needed, Pistorius studies the (A, B) exit (A, A) entrance, and we find (A, B) exit (B, A) entrance. Spectral positivity disallows B exit at 0 and $\mathrm{B}$ entrance from $b$, but this leaves a number of other possibilities, in principle, which we do not pursue further.

Scale functions $W^{(q)}$ with Laplace transform $\int_{0}^{\infty} e^{-\eta x} W^{(q)}(x) d x=1 /(\psi(\eta)-$ $q)$ and $Z^{(q)}(x)=1+q \int_{0}^{x} W^{(q)}(z) d z$ are well-known functions in the fluctuation theory of spectrally one-sided Lévy processes; see, for example, [38].

Proposition 21. The Laplace exponent of the inverse local time $\sigma^{b}$ of $X^{b}$ at 0 is given by

$$
\begin{aligned}
\Theta^{b}(q) & =-\log \left(\mathbb{E}\left(\exp \left(-q \sigma_{1}^{b}\right)\right)\right) \\
& =\int_{0}^{b}\left(1-\frac{Z^{(q)}(b-x)}{Z^{(q)}(b)}\right) \chi(d x)+\left(1-\frac{1}{Z^{(q)}(b)}\right) \chi((b, \infty)) \\
& =\frac{1}{Z^{(q)}(b)} \int_{0}^{b} \overline{\bar{\Pi}}(b-z) q W^{(q)}(z) d z,
\end{aligned}
$$

where $\overline{\bar{\Pi}}(x)=\int_{x}^{\infty} \bar{\Pi}(z) d z$. Furthermore, $\sigma^{b}$ has moments of all orders.

Proof. First, note that $\overline{\bar{\Pi}}(x)<\infty$ for all $x>0$ since $X$ has finite (zero) mean. After the discussion preceding the proposition, the result follows directly from the exponential formula for the Poisson point process of excursions away from zero and the first exit problem of the reflected process $\widehat{X}^{+}$from $(0, b)$. Specifically, $T_{\geq 0}(\omega)=0$ for $n^{b}$-a.e. $\omega$, and the post- $T_{\geq 0}$ process under the excursion measure $n^{\bar{b}}$ of $X^{b}$ away from 0 is the post- $T_{\geq 0}$ process of the restricted process absorbed at 0 , which is the same as $b$ minus the reflected process $\widehat{X}^{+}$absorbed at $b$. By [39], Proposition 2,

$$
\mathbb{E}_{x}\left(e^{-q T_{0}\left(X^{b}\right)}\right)=\frac{Z^{(q)}(b-x)}{Z^{(q)}(b)}, \quad x \in(0, b],
$$


for $x>b$, the post- $T_{\geq 0}$ process under $n^{b}$ starts from $b$. Let $W=W^{(0)}$ and recall, for example, from [6]

$$
\begin{aligned}
W^{(q)}(x)= & \sum_{k \geq 0} q^{k} W^{*(k+1)}(x), \\
& \text { where for the convolution } W^{*(k+1)}(x) \leq \frac{1}{k !} x^{k} W^{(0)}(x)^{k+1},
\end{aligned}
$$

so that $q \mapsto W^{(q)}(x)$ is analytic on $\mathbb{C}$. Hence, we calculate by (12) and using Fubini's theorem

$$
\begin{aligned}
\Theta^{b}(q)= & -\log \left(\mathbb{E}\left(\exp \left(-q \sigma_{1}^{b}\right)\right)\right) \\
= & \int_{0}^{b}\left(1-\frac{Z^{(q)}(b-x)}{Z^{(q)}(b)}\right) \chi(d x)+\left(1-\frac{1}{Z^{(q)}(b)}\right) \chi((b, \infty)) \\
= & \frac{1}{Z^{(q)}(b)}\left(\int_{0}^{b} \int_{b-x}^{b} q W^{(q)}(z) d z \bar{\Pi}(x) d x\right. \\
& \left.+\int_{0}^{b} q W^{(q)}(z) d z \int_{b}^{\infty} \bar{\Pi}(x) d x\right) \\
= & \frac{1}{Z^{(q)}(b)} \int_{0}^{b} \overline{\bar{\Pi}}(b-z) q W^{(q)}(z) d z \\
= & \frac{1}{Z^{(q)}(b)} \int_{0}^{b} \int_{b-z}^{\infty} \bar{\Pi}(x) d x q \sum_{k \geq 0} q^{k} W^{*(k+1)}(z) d z \\
= & \frac{1}{Z^{(q)}(b)} \sum_{j \geq 1} q^{j} \int_{0}^{b} \overline{\bar{\Pi}}(b-z) W^{*(j)}(z) d z .
\end{aligned}
$$

Now $\Theta^{b}(q)$ is a ratio of complex power series, hence infinitely differentiable within the radius where the (analytic) denominator is nonzero. Since $Z^{(q)}(0)=1$, all moments of $\sigma^{b}$ are finite.

COROLlary 22. If $X$ is a spectrally positive stable process with Laplace exponent $\psi(\eta)=a \eta^{1+\alpha}$ for some $a>0$ and $1+\alpha \in(1,2)$, then

$$
\Theta^{b}(q)=\frac{b^{-\alpha}}{E_{1+\alpha}\left(q b^{1+\alpha} / a\right)}\left(E_{1+\alpha, 1-\alpha}\left(q b^{1+\alpha} / a\right)-\frac{1}{\Gamma(1-\alpha)}\right),
$$

where $E_{1+\alpha, \beta}(x)=\sum_{k \geq 0} x^{k} / \Gamma(\beta+k(1+\alpha))$ is the two-parameter MittagLeffler function, a complex-analytic function on all of $\mathbb{C}$ for all $\beta>0$, and where $E_{1+\alpha}=E_{1+\alpha, 1}$. 
PROOF. Since varying $a$ corresponds to a linear time change of $X$, we can assume $a=1$ w.l.o.g. By [5], $Z^{(q)}(b)=E_{1+\alpha}\left(q b^{1+\alpha}\right)$. Also

$$
\bar{\Pi}(b)=\int_{b}^{\infty} \frac{(1+\alpha) \alpha}{\Gamma(1-\alpha)} x^{-\alpha-2} d x=\frac{\alpha}{\Gamma(1-\alpha)} b^{-\alpha-1} .
$$

With $\chi(d x)=\bar{\Pi}(x) d x$, from (12),

$$
\begin{aligned}
\Theta^{b}(q)= & \frac{1}{Z^{(q)}(b)}\left(\int_{0}^{b}\left(Z^{(q)}(b)-Z^{(q)}(b-x)\right) \chi(d x)\right. \\
& \left.+\left(Z^{(q)}(b)-1\right) \chi((b, \infty))\right) \\
= & {\left[\alpha \int_{0}^{b}\left(E_{1+\alpha}\left(q b^{1+\alpha}\right)-E_{1+\alpha}\left(q(b-x)^{1+\alpha}\right)\right) x^{-\alpha-1} d x\right.} \\
& \left.+b^{-\alpha}\left(E_{1+\alpha}\left(q b^{1+\alpha}\right)-1\right)\right] \times\left[Z^{(q)}(b) \Gamma(1-\alpha)\right]^{-1} .
\end{aligned}
$$

We use power series techniques to calculate the first term of the numerator:

$$
\begin{aligned}
\int_{0}^{b} & \left(E_{1+\alpha}\left(q b^{1+\alpha}\right)-E_{1+\alpha}\left(q(b-x)^{1+\alpha}\right)\right) x^{-\alpha-1} d x \\
& =\sum_{k=1}^{\infty} \frac{q^{k}}{\Gamma(1+k(1+\alpha))} \int_{0}^{b}\left(b^{k(1+\alpha)}-(b-x)^{k(1+\alpha)}\right) x^{-\alpha-1} d x .
\end{aligned}
$$

We now solve this integral using Fubini theorem and Beta integrals to find finite coefficients

$$
\begin{aligned}
& \frac{1}{\Gamma(1+k(1+\alpha))} \int_{0}^{b}\left(b^{k(1+\alpha)}-(b-x)^{k(1+\alpha)}\right) x^{-\alpha-1} d x \\
& =\frac{b^{k(1+\alpha)-\alpha}}{\Gamma(1+k(1+\alpha))} \frac{1}{\alpha}\left(\frac{\Gamma(1-\alpha) \Gamma(k(1+\alpha)+1)}{\Gamma(k(1+\alpha)+1-\alpha)}-1\right) .
\end{aligned}
$$

The first term gives coefficients of the two-parameter Mittag-Leffler function. The second term gives coefficients just as needed to cancel with most of the second term in the numerator of (13); we obtain the formula we claimed for $a=1$. To pass to general $a>0$, it is easy to check that $Z^{(q)}(b)=E_{1+\alpha}\left(q b^{1+\alpha} / a\right)$, so we can just replace $q$ by $q / a$.

\subsection{Uniform local time estimates up to random times.}

PROPOSITION 23. Consider a spectrally positive stable Lévy process $X$ with Laplace exponent $\eta^{1+\alpha}$. There is $K>0$ such that for all $x, y \in \mathbb{R}$ and all $N, r \in$ $(0, \infty)$ we have

$$
\mathbb{P}\left(\sup _{0 \leq t \leq N}\left|\ell^{y}(t)-\ell^{x}(t)\right|>r\right) \leq 2 e^{N} \exp \left(-K r|y-x|^{-\alpha / 2}\right) .
$$


Hence for all $p>0$, we also have $\mathbb{E}\left(\sup _{0 \leq t \leq N}\left|\ell^{y}(t)-\ell^{x}(t)\right|^{p}\right) \leq 2 \Gamma(p+$ 1) $K^{-p} e^{N}|y-x|^{p \alpha / 2}$.

LEMma 24. For $z>0$, let $\varphi(z) \in(0,1)$ be such that we have $(\varphi(z))^{2}=1-$ $\Psi_{0}(z) \Psi_{z}(0)$, where $\Psi_{x}(y)=\mathbb{E}_{x}\left(\exp \left(-T_{y}(X)\right)\right)$ and $T_{y}(X)=\inf \left\{t \geq 0: X_{t}=y\right\}$. Then there is $K>0$ such that $2 \varphi(z) \leq z^{\alpha / 2} / K$ for all $z>0$.

PROOF. We may assume that $z \leq 1$ since the bound is trivially true for $z \geq 1$ provided $K \leq 1$. Note that $\lim _{y \rightarrow 0} T_{y}(X)=0$ almost surely under $\mathbb{P}_{0}$ and, consequently, Lemma 12 and the dominated convergence theorem combine to imply that for $\lambda \sim \operatorname{Exp}(1)$,

$$
1=\lim _{y \downarrow 0} \mathbb{P}\left(T_{y}(X)<\lambda\right)=\frac{1+\alpha}{\pi} \int_{0}^{\infty} \frac{\sin (\pi \alpha) s^{1+\alpha}}{s^{2(1+\alpha)}+2 s^{1+\alpha} \cos (\pi \alpha)+1} d s .
$$

Also recall from Lemma 12 that $\Psi_{z}(0)=\mathbb{E}_{0}\left(e^{-T_{z}(X)}\right)=e^{-z}$. Consequently,

$$
\begin{aligned}
(\varphi(z))^{2}= & 1-\Psi_{0}(z) \Psi_{z}(0) \\
= & 1-\exp (-z) \frac{1+\alpha}{\pi} \int_{0}^{\infty} \frac{\sin (\pi \alpha) s^{1+\alpha}}{s^{2(1+\alpha)}+2 s^{1+\alpha} \cos (\pi \alpha)+1} e^{-z s} d s \\
= & \frac{1+\alpha}{\pi} \int_{0}^{\infty} \frac{\sin (\pi \alpha) s^{1+\alpha}}{s^{2(1+\alpha)}+2 s^{1+\alpha} \cos (\pi \alpha)+1}\left(1-e^{-(s+1) z}\right) d s \\
\leq & \frac{(1+\alpha) \sin (\pi \alpha)}{\pi} z \int_{0}^{z^{-1}} \frac{s^{1+\alpha}}{s^{2(1+\alpha)}+2 s^{1+\alpha} \cos (\pi \alpha)+1}(1+s) d s \\
& +\frac{(1+\alpha) \sin (\pi \alpha)}{\pi} \int_{z^{-1}}^{\infty} \frac{s^{1+\alpha}}{s^{2(1+\alpha)}+2 s^{1+\alpha} \cos (\pi \alpha)+1} d s .
\end{aligned}
$$

Splitting $s^{2(1+\alpha)}=s^{2(1+\alpha)} \sin ^{2}(\pi \alpha)+s^{2(1+\alpha)} \cos ^{2}(\pi \alpha)$, we can further bound above by

$$
\begin{gathered}
z+\frac{1+\alpha}{\pi \sin (\pi \alpha)} z \int_{0}^{z^{-1}} s^{-\alpha} d s+\frac{(1+\alpha)}{\pi \sin (\pi \alpha)} \int_{z^{-1}}^{\infty} s^{-1-\alpha} d s \\
=z+\frac{1+\alpha}{(1-\alpha) \pi \sin (\pi \alpha)} z^{\alpha}+\frac{(1+\alpha)}{\alpha \pi \sin (\pi \alpha)} z^{\alpha} \\
\leq z^{\alpha}\left(1+\frac{1+\alpha}{(1-\alpha) \pi \sin (\pi \alpha)}+\frac{(1+\alpha)}{\alpha \pi \sin (\pi \alpha)}\right),
\end{gathered}
$$

and the result follows.

Proof of Proposition 23. By [8], Proposition V.(3.28), we have for all $N, \delta \in(0, \infty)$ and $x, y \in \mathbb{R}$

$$
\mathbb{P}\left(\sup _{0 \leq t \leq N}\left|\ell^{y}(t)-\ell^{x}(t)\right|>2 \delta\right) \leq 2 e^{N} \exp (-\delta / \varphi(|y-x|)),
$$


where $\varphi$ is as in Lemma 24, and setting $r=2 \delta, z=|y-x|$ and with $K$ as in the preceding lemma, we conclude for the first inequality. The second is an elementary integration of the first.

In fact, the statement on moments, when expressed in terms of $\varphi$, also holds in the full generality of the Blumenthal-Getoor result, for their notion of a standard Markov process. Since the tail estimate is also independent of the starting value of $X$, by the strong Markov property at $T_{x} \wedge T_{y}$, it turns out that it is easy to improve the dependence on $N$ in the moment bounds, in the general case. In particular, we obtain the following.

COROLlaRY 25. There is $K>0$, such that for all $p>0, x, y \in \mathbb{R}$ and $N>0$,

$$
\mathbb{E}\left(\sup _{0 \leq t \leq N}\left|\ell^{y}(t)-\ell^{x}(t)\right|^{p}\right) \leq 2 \Gamma(p+1) K^{-p}(N+1)^{p+1} e|y-x|^{p \alpha / 2} .
$$

Proof. Writing $\ell^{y}(t)$ and $\ell^{x}(t)$ as sums of increments $\ell^{\cdot}(k+1)-\ell^{\cdot}(k)$ and $\ell \cdot(t)-\ell^{\cdot}(\lfloor t\rfloor)$, we find

$$
\begin{aligned}
\sup _{0 \leq t \leq N} & \left|\ell^{y}(t)-\ell^{x}(t)\right| \\
& \leq \sum_{0 \leq k \leq\lfloor N\rfloor} \sup _{0 \leq s \leq 1}\left|\left(\ell^{y}(k+s)-\ell^{y}(k)\right)-\left(\ell^{x}(k+s)-\ell^{x}(k)\right)\right| .
\end{aligned}
$$

We estimate this sum as follows:

$$
\left(\sum_{k=0}^{\lfloor N\rfloor} x_{k}\right)^{p} \leq\left((N+1) \max _{0 \leq k \leq\lfloor N\rfloor} x_{k}\right)^{p} \leq(N+1)^{p} \sum_{k=0}^{\lfloor N\rfloor} x_{k}^{p}
$$

By the Markov property and since local times are additive functionals, all $\lfloor N\rfloor+1$ terms satisfy the same moment bound from Proposition 23, applied for unit time intervals, so we obtain the estimate as claimed.

COROLLARY 26. For any random time $V$ with moments of all orders and any $p>0$, there is $M_{p}$ depending on the distribution of $V$ such that for all $x, y \in \mathbb{R}$, we have

$$
\mathbb{E}\left(\sup _{0 \leq t \leq V}\left|\ell^{y}(t)-\ell^{x}(t)\right|^{p}\right) \leq M_{p}|y-x|^{p \alpha / 2}
$$

PROOF. We use the Cauchy-Schwarz inequality to obtain

$$
\begin{aligned}
& \mathbb{E}\left(\sup _{0 \leq t \leq V}\left|\ell^{y}(t)-\ell^{x}(t)\right|^{p}\right) \\
& \quad \leq \sum_{k \geq 2} \mathbb{E}\left(1_{\{k-2 \leq V<k-1\}} \sup _{0 \leq t \leq k-1}\left|\ell^{y}(t)-\ell^{x}(t)\right|^{p}\right)
\end{aligned}
$$




$$
\begin{aligned}
& \leq \sum_{k \geq 2}(\mathbb{P}(k-2 \leq V<k-1))^{1 / 2}\left(\mathbb{E}\left(\sup _{0 \leq t \leq k-1}\left|\ell^{y}(t)-\ell^{x}(t)\right|^{2 p}\right)\right)^{1 / 2} \\
& \leq\left(\sum_{k \geq 2} k^{p+1 / 2}(\mathbb{P}(V \geq k-2))^{1 / 2}\right) \sqrt{2 e \Gamma(2 p+1)} K^{-p}|y-x|^{p \alpha / 2} .
\end{aligned}
$$

Since finite $p^{\prime}$ th moment of $V$ implies that there is $k_{0}\left(p^{\prime}\right) \geq 1$ such that $\mathbb{P}(V \geq$ $k-2) \leq k^{-p^{\prime}}$ for all $k \geq k_{0}\left(p^{\prime}\right)$, we can choose any $p^{\prime}>2 p+3$ to see that the series in our expression converges.

Now consider the spectrally positive stable- $(1+\alpha)$ process restricted to an interval $[0, b]$. Recall that this process is defined as $X_{r}^{b}=X_{\tau^{[0, b]}(r)}$, where

$$
\tau^{[0, b]}(r)=\inf \left\{t \geq 0: R^{[0, b]}(t)>r\right\} \quad \text { and } \quad R^{[0, b]}(t)=\int_{0}^{t} 1_{\left\{0 \leq X_{s} \leq b\right\}} d s .
$$

Suppose we are only interested in $\mathbb{E}\left(\sup _{0 \leq t \leq V}\left|\ell^{y}(t)-\ell^{x}(t)\right|^{p}\right)$ for a restricted range of $x, y \in[a, b]$. We can use the fact that $X^{b}$ is a Markov process with the same local time differences as $X$ in the interval $[0, b]$ to improve on this corollary and effectively allow times $V=\tau^{[0, b]}(Q)$, where only $Q$ is required to have moments of all orders. This is a genuine improvement because $\mathbb{E}\left(\tau^{[0, b]}(r)\right)=\infty$ for all $r \geq 0$, since there is positive probability that $X$ leaves $(-\infty, b]$ during $[0, r]$ after which to return inside $[0, b]$ it takes $X$ a level passage time given by a stable$(1 /(1+\alpha))$ ladder time subordinator.

PROPOSITION 27. For any $b>0$, any random time $Q$ with moments of all orders and any $p>0$, there is $M_{p, b}$ depending on the distribution of $Q$ such that for all $x, y \in[0, b]$, we have

$$
\mathbb{E}\left(\sup _{0 \leq t \leq \tau^{[0, b]}(Q)}\left|\ell^{y}(t)-\ell^{x}(t)\right|^{p}\right) \leq M_{p, b}|y-x|^{p \alpha / 2} .
$$

PROOF. We repeat some of the previous arguments. First, [8], Proposition V.(3.28), applies to $X^{b}$ with local times $\ell_{b}^{x}(r)=\ell^{x}\left(\tau^{[0, b]}(r)\right), 0 \leq x \leq b, r \geq 0$ : for all $N>0, \delta>0, x, y, z \in[0, b]$,

$$
\mathbb{P}_{z}\left(\sup _{0 \leq t \leq N}\left|\ell_{b}^{y}(r)-\ell_{b}^{x}(r)\right| \geq 2 \delta\right) \leq 2 e^{N} \exp \left(-\delta / 2 \varphi_{b}(x, y)\right),
$$

where

$$
\begin{aligned}
\left(\varphi_{b}(x, y)\right)^{2} & =1-\mathbb{E}_{x}\left(\exp \left(-T_{y}\left(X^{b}\right)\right)\right) \mathbb{E}_{y}\left(\exp \left(-T_{x}\left(X^{b}\right)\right)\right) \\
& \leq 1-\mathbb{E}_{x}\left(\exp \left(-T_{y}(X)\right)\right) \mathbb{E}_{y}\left(\exp \left(-T_{x}(X)\right)\right)=(\varphi(|y-x|))^{2} .
\end{aligned}
$$

By Lemma 24 and integration, we find $K>0$ such that for all $p>0$,

$$
\mathbb{E}_{z}\left(\sup _{0 \leq r \leq N}\left|\ell_{b}^{y}(r)-\ell_{b}^{x}(r)\right|^{p}\right) \leq 2 \Gamma(p+1) K^{-p} e^{N}|y-x|^{p \alpha / 2} .
$$


Now the arguments of Corollaries 25 and 26 apply to give $M_{p, b}$ so that

$$
\begin{aligned}
\mathbb{E}\left(\sup _{0 \leq t \leq \tau^{[0, b]}(Q)}\left|\ell^{y}(t)-\ell^{x}(t)\right|^{p}\right) & =\mathbb{E}\left(\sup _{0 \leq r \leq Q}\left|\ell_{b}^{y}(t)-\ell_{b}^{x}(t)\right|^{p}\right) \\
& \leq M_{p, b}|y-x|^{p \alpha / 2} .
\end{aligned}
$$

THEOREM 28. For any $b>0$, any random time $Q$ with moments of all orders and any $v \in(0, \alpha / 2)$, the random variable

$$
D_{v}^{b}\left(\tau^{[0, b]}(Q)\right)=\sup _{0 \leq t \leq \tau^{[0, b]}(Q), 0 \leq x<y \leq b} \frac{\left|\ell^{y}(t)-\ell^{x}(t)\right|}{|y-x|^{v}}<\infty \quad \text { a.s. }
$$

has moments of all orders.

PROOF. Now consider the Banach space of bounded continuous functions from $[0, \infty)$ to $\mathbb{R}$ equipped with the supremum norm $\|\cdot\|_{\infty}$ (rather than any localised version). Then the stopped local time processes $L_{v}=\left(\ell^{b v}\left(t \wedge \tau^{[0, b]}(Q)\right)\right.$, $t \geq 0$ ), with levels $[0, b]$ parametrised as $b v, v \in[0,1]$, are members of this Banach space and satisfy

$$
\mathbb{E}\left(\left\|L_{v}-L_{u}\right\|_{\infty}^{p}\right) \leq M_{p, b} b^{p \alpha / 2}|v-u|^{p \alpha / 2} \quad \text { for all } u, v \in[0,1],
$$

by Proposition 27. We can apply the Revuz-Yor version of the KolmogorovChentsov theorem [43], Theorem I.(2.1), with $\varepsilon=p \alpha / 2-1$, to find that

$$
\mathbb{E}\left(\left(D_{v}^{b}\left(\tau^{[0, b]}(Q)\right)\right)^{p}\right)=\frac{1}{b^{p v}} \mathbb{E}\left(\left(\sup _{0 \leq u<v \leq 1} \frac{\left\|L_{v}-L_{u}\right\|_{\infty}}{|v-u|^{v}}\right)^{p}\right)<\infty,
$$

as long as $v \in(0, \varepsilon / p)$, that is, if $v<(\alpha / 2)-(1 / p)$, which gives the result for $v \in(0, \alpha / 2)$ by letting $p \rightarrow \infty$.

Now consider the Poisson point process of excursions of $X$ away from 0 , enriched by marking all jumps by paths according to a nontrivial marking kernel. Further mark each excursion with probability $1-e^{-m}$, where $m$ is the value of the path in the jump of $X$ across 0 when crossing 0 . Denote by $S$ the time at which the excursion point process has the first mark, and by $T$ the left endpoint of the corresponding excursion of $X$.

COROllary 29. For any $b>0$ and $v \in(0, \alpha / 2)$, the random variable

$$
D_{v}^{[0, b]}:=D_{v}^{[0, b]}(T)=\sup _{0 \leq t \leq T, 0 \leq x<y \leq b} \frac{\left|\ell^{y}(t)-\ell^{x}(t)\right|}{|y-x|^{v}}<\infty \quad \text { a.s. }
$$

and has moments of all orders. 
Proof. First, note that $T=\tau^{[0, b]}\left(\sigma_{S-}^{b}\right) \leq \tau^{[0, b]}\left(\sigma_{S}^{b}\right)$. By elementary properties of Poisson point processes, $S$ is exponentially distributed, with parameter $\mu$, say. Note, however, that $S$ is not independent of $\sigma^{b}$. We use the same argument as in Corollary 26 and (14) to find

$$
\begin{aligned}
\mathbb{E}\left(\left(\sigma_{S}^{b}\right)^{p}\right) & \leq \sum_{k \geq 1}(\mathbb{P}(S \geq k-1))^{1 / 2}\left(\mathbb{E}\left(\left(\sigma_{k}^{b}\right)^{2 p}\right)\right)^{1 / 2} \\
& \leq\left(\sum_{k \geq 1} e^{-\mu k / 2} k^{p+1 / 2}\right) e^{\mu / 2}\left(\mathbb{E}\left(\left(\sigma_{1}^{b}\right)^{2 p}\right)\right)^{1 / 2} .
\end{aligned}
$$

By Proposition 21, the inverse local time $\sigma_{1}^{b}$ of $X^{b}$ has moments of all orders. Also the series clearly converges. Hence, $\sigma_{S}^{b}$ has moments of all orders, as does $\sigma_{S-}^{b}<\sigma_{S}^{b}$. Hence, Theorem 28 applies to $Q=\sigma_{S-}^{b}$, and this completes the proof.

\subsection{Proof of Theorem 3.}

Proof of THEOREM 3. We repeat the argument of the previous proof. Specifically, we apply Theorem 28 to $Q=R^{[0, b]}\left(\tau^{0}(S)\right)=\sigma_{S}^{b}$.

6. Moments of Hölder constants of BESQ processes, bridges and excursions. In this section, we study examples of paths $Z$ as needed for Theorem 1, specifically, Brownian paths and squared Bessel processes (BESQ) of dimension $\delta \in \mathbb{R}$. We check that they satisfy the moment assumption of Theorem 1 . Section 6.1 studies BESQ processes as sums of i.i.d. squared Brownian motions. Section 6.2 uses stochastic calculus and captures a larger class of BESQ-type stochastic differential equations. We specialise to BESQ processes in Section 6.3. Section 6.4 demonstrates how Theorem 1 can be applied when jumps are marked by BESQ excursions. This model plays an important role in [19].

\subsection{Brownian motion, Brownian bridge and Brownian excursion.}

LEMMA 30. Let $\left(B_{t}, 0 \leq t \leq 1\right)$ be standard Brownian motion and $\gamma \in$ $(0,1 / 2)$. Then

$$
D_{\gamma}=\sup _{0 \leq s<t \leq 1} \frac{\left|B_{t}-B_{s}\right|}{|t-s|^{\gamma}}<\infty \quad \text { a.s. }
$$

and the uniform bound $D_{\gamma}$ has moments of all orders.

PROOF. This is well known and follows straight from the KolmogorovChentsov theorem; see, for example, Revuz and Yor [43], Theorem I.(2.1). Specifically, by scaling, we have $\mathbb{E}\left(\left|B_{t}-B_{s}\right|^{2 p}\right)=C_{p}|t-s|^{p}$ for all $p>0$, so the 
theorem yields $\mathbb{E}\left(D_{\gamma}^{p}\right)<\infty$ as long as $p>1$ and $0<\gamma<1 / 2-1 /(2 p)$, that is, $0<\gamma<1 / 2$ and $p>1 /(1-2 \gamma)$. This gives moments of all orders, as required.

COROLlaRY 31. Let $\left(B_{t}^{\mathrm{br}}, 0 \leq t \leq 1\right)$ and $\left(B_{t}^{\mathrm{ex}}, 0 \leq t \leq 1\right)$ be standard Brownian bridge and standard Brownian excursion and $\gamma \in(0,1 / 2)$. Then

$D_{\gamma}^{\mathrm{br}}=\sup _{0 \leq s<t \leq 1} \frac{\left|B_{t}^{\mathrm{br}}-B_{s}^{\mathrm{br}}\right|}{|t-s|^{\gamma}}<\infty \quad$ and $\quad D_{\gamma}^{\mathrm{ex}}=\sup _{0 \leq s<t \leq 1} \frac{\left|B_{t}^{\mathrm{ex}}-B_{s}^{\mathrm{ex}}\right|}{|t-s|^{\gamma}}<\infty \quad$ a.s.

and the uniform bounds $D_{\gamma}^{\mathrm{br}}$ and $D_{\gamma}^{\mathrm{ex}}$ have moments of all orders.

PROOF. We use the pathwise representations, due to Lévy and Vervaat:

$$
B_{t}^{\mathrm{br}}=B_{t}-t B_{1}, \quad 0 \leq t \leq 1 \quad \text { and } \quad B_{t}^{\mathrm{ex}}= \begin{cases}B_{M+t}^{\mathrm{br}}, & 0 \leq t \leq 1-M, \\ B_{t-(1-M)}, & 1-M \leq t \leq 1,\end{cases}
$$

where $M=\inf \left\{t \geq 0: B_{t}^{\mathrm{br}}=\min \left\{B_{r}^{\mathrm{br}}, 0 \leq r \leq 1\right\}\right\}$. Then we have for all $0 \leq s<$ $t \leq 1$

$$
\left|B_{t}^{\mathrm{br}}-B_{s}^{\mathrm{br}}\right| \leq\left|B_{t}-B_{s}\right|+|t-s|\left|B_{1}-B_{0}\right| \leq 2 D_{\gamma}|t-s|^{\gamma}
$$

and similarly for $\left|B_{t}^{\mathrm{ex}}-B_{s}^{\mathrm{ex}}\right|$ if $0 \leq s<t \leq 1-M$ or $1-M \leq s<t \leq 1$. For $0 \leq s<1-M<t \leq 1$,

$$
\begin{aligned}
\left|B_{t}^{\mathrm{ex}}-B_{s}^{\mathrm{ex}}\right| & =\left|B_{M+s}^{\mathrm{br}}-B_{t-(1-M)}^{\mathrm{br}}\right| \\
& \leq\left|B_{M+s}^{\mathrm{br}}-B_{1}^{\mathrm{br}}\right|+\left|B_{t-(1-M)}^{\mathrm{br}}-B_{0}^{\mathrm{br}}\right| \\
& \leq 4 D_{\gamma}|t-s|^{\gamma} .
\end{aligned}
$$

Hence, $D_{\gamma}^{\mathrm{br}} \leq 2 D_{\gamma}$ and $D_{\gamma}^{\mathrm{ex}} \leq 4 D_{\gamma}$.

$$
\text { Via }\left|\left(B_{t}^{\mathrm{br}}\right)^{2}-\left(B_{s}^{\mathrm{br}}\right)^{2}\right|=\left|B_{t}^{\mathrm{br}}-B_{s}^{\mathrm{br}}\right|\left|B_{t}^{\mathrm{br}}+B_{s}^{\mathrm{br}}\right| \leq 4 D_{\gamma}^{\mathrm{br}} \sup _{0 \leq r \leq 1}\left|B_{r}^{\mathrm{br}}\right| \text {, Cauchy- }
$$
Schwarz (or binomial formulas), the fact that $\sup _{0 \leq r \leq 1}\left|B_{r}^{\mathrm{br}}\right| \leq \sup _{0 \leq r \leq 1}\left|B_{r}^{\mathrm{ex}}\right|$ in the Vervaat coupling and the fact that the maximum of $B^{\mathrm{ex}}$ has moments of all orders, these results extends to BESQ(1)-bridges. By [41], Remark (5.8)(i), on the one hand sums of $\operatorname{BESQ}(1)$ bridges are $\operatorname{BESQ}(\delta)$ bridges for $\delta \in \mathbb{N}$, while suitable couplings of noninteger dimensions allow to extend this to any real dimension $\delta \geq 1$, and on the other hand, these bridges are also the normalised $\operatorname{BESQ}(4-$ $\delta$ ) excursions, so all these processes have Hölder constants with moments of all orders.

We will use a different approach to also include results for BESQ processes starting from $x \neq 0$. Such processes appear in the present paper, as well as further work in [19], as the processes $\left(Z_{t}\left(y-X_{t-}\right), y \geq 0\right)$, when $X_{t-}<0$. In the study of the process $Z_{[0, T]}(y)=\sum_{0 \leq t \leq T} Z_{t}\left(y-X_{t-}\right), y \geq 0$ of (1), these populations already have a positive mass at level 0 , and we may be interested in conditioning on the initial population; see [19] for more details. 
6.2. Results for general diffusions of BESQ-type. Our arguments are adapted from those by Hutzenthaler et al. [23], where similar results are obtained for a different class of processes.

LEMMA 32. Let $\left(X_{t}, t \geq 0\right)$ be a $\operatorname{BESQ}(\delta)$ process starting from $x \geq 0$, for some dimension parameter $\delta \geq 0$. Then

$$
\left\|X_{t}\right\|_{p} \leq x+t\left(\delta+2(p-1)^{+}\right) \quad \text { for all } t \geq 0 \text { and } p \in(0, \infty) .
$$

PROOF. Recall, for example, from [22], Definition 1, that we may assume that for a Brownian motion $B$ :

$$
X_{t}=x+\delta t+2 \int_{0}^{t} \sqrt{X_{s}} d B_{s} \quad \text { a.s., } \quad t \geq 0 .
$$

By continuity of $p$-norms, it suffices to show that for all $\varepsilon \in(0,1)$ and $k \geq 0$,

(16) $\left\|X_{t}+\varepsilon\right\|_{p} \leq x+\varepsilon+t\left(\delta+2(p-1)^{+}\right) \quad$ for all $t \geq 0$ and $p \in(k, k+1]$.

We will prove this by induction on $k$. For $k=0$ note that by (15)

$$
\begin{aligned}
\mathbb{E}\left(X_{t}\right) & =x+\delta t \\
& \Rightarrow\left(\mathbb{E}\left(\left(X_{t}+\varepsilon\right)^{p}\right)\right)^{1 / p} \leq \mathbb{E}\left(X_{t}+\varepsilon\right)=x+\varepsilon+t \delta \quad \text { for all } p \in(0,1] .
\end{aligned}
$$

Assuming (16) for some $k \geq 0$, we obtain for all $t \geq 0$ and $p \in(k+1, k+2]$ by Itô's lemma that

$$
\begin{aligned}
& \mathbb{E}\left(\left(X_{t}+\varepsilon\right)^{p}\right) \\
&=(x+\varepsilon)^{p}+\int_{0}^{t}\left(\delta p \mathbb{E}\left(\left(X_{s}+\varepsilon\right)^{p-1}\right)\right. \\
&\left.\quad+2 p(p-1) \mathbb{E}\left(X_{s}\left(X_{s}+\varepsilon\right)^{p-2}\right)\right) d s \\
& \leq(x+\varepsilon)^{p}+\int_{0}^{t} p(\delta+2(p-1)) \mathbb{E}\left(\left(X_{s}+\varepsilon\right)^{p-1}\right) d s \\
& \leq(x+\varepsilon)^{p}+\int_{0}^{t} p(\delta+2(p-1))\left(x+\varepsilon+s\left(\delta+2(p-2)^{+}\right)\right)^{p-1} d s \\
& \leq(x+\varepsilon)^{p}+(x+\varepsilon+t(\delta+2(p-1)))^{p}-(x+\varepsilon)^{p} \\
&=(x+\varepsilon+t(\delta+2(p-1)))^{p} .
\end{aligned}
$$

LEMMA 33. Let $\mu: \mathbb{R} \rightarrow \mathbb{R}$ be Lipschitz and bounded by $c>0$. Let $\left(B_{t}, t \geq\right.$ $0)$ be Brownian motion. For $x \in \mathbb{R}$, let $\left(X_{t}, t \geq 0\right)$ be a stochastic process with continuous sample paths adapted to the same filtration as $B$ and satisfying $\int_{0}^{t}\left|\mu\left(X_{s}\right)\right| d s<\infty$ a.s. and

$$
X_{t}=x+\int_{0}^{t} \mu\left(X_{r}\right) d r+2 \int_{0}^{t} \sqrt{\left|X_{r}\right|} d B_{r} \quad \text { a.s., } t \geq 0 .
$$


Then for all $t \geq s \geq 0$ and $p \in[2, \infty)$ we have that

$$
\begin{aligned}
\left\|X_{t}-X_{s}\right\|_{p} \leq & \sqrt{t-s}(\sqrt{t} c+\sqrt{t} 2 \sqrt{(p-1)(c+p-2)} \\
& +2 \sqrt{p-1} \sqrt{|x|+2 t(c+p-2)}) .
\end{aligned}
$$

We remark that for any $\mu$ satisfying the hypotheses of the lemma, the weak existence and pathwise uniqueness hold for the SDE (13) by [18], Theorem 5.3.8 and Theorem 5.3.10. Strong existence of solutions and the Markov property of solutions then follows from [26], Theorem 21.11 and Theorem 21.14.

PRoof. By passing to $-X$ if needed, we may assume that $x \geq 0$. Using the strong existence of solutions to the squared Bessel process SDE and the comparison of one-dimensional diffusions, there exists a BESQ $(c)$ process $\left(Z_{t}\right)_{t \geq 0}$ starting from $x$ and a $\mathrm{BESQ}(-c)$ process $\left(Y_{t}\right)_{t \geq 0}$ starting from 0 such that $Y_{t} \leq X_{t} \leq Z_{t}$ for all $t \geq 0$. Since $Y_{t} \leq 0$ and $Z_{t} \geq 0$, we have that $\left|X_{t}\right|^{p} \leq Z_{t}^{p}+\left|Y_{t}\right|^{p}$. Note that $\left|Y_{t}\right|$ is a $\operatorname{BESQ}(c)$ process starting from 0 . Using the inequality $(x+y)^{1 / p} \leq$ $x^{1 / p}+y^{1 / p}$ for $x, y>0$ and $p \geq 1$, Lemma 32 implies that

$$
\left\|X_{t}\right\|_{p} \leq\left\|Z_{t}\right\|_{p}+\left\|Y_{t}\right\|_{p} \leq|x|+2 t(c+2(p-1)) .
$$

Now consider the case $s=0$. Then

$$
\begin{aligned}
\left\|X_{t}-x\right\|_{p} & \leq\left\|\int_{0}^{t}\left|\mu\left(X_{r}\right)\right| d r\right\|_{p}+\left\|\int_{0}^{t} 2 \sqrt{\left|X_{r}\right|} d B_{r}\right\|_{p} \\
& \leq c t+\left\|\int_{0}^{t} 2 \sqrt{\left|X_{r}\right|} d B_{r}\right\|_{p} .
\end{aligned}
$$

Let $M_{t}=\int_{0}^{t} 2 \sqrt{\left|X_{r}\right|} d B_{r}$. Since $p \geq 2$, Itô's lemma implies

$$
\begin{aligned}
M_{t}^{p} & =\int_{0}^{t} p M_{r}^{p-1} d M_{r}+\frac{p(p-1)}{2} \int_{0}^{t} M_{r}^{p-2} d[M]_{r} \\
& =\int_{0}^{t} p M_{r}^{p-1} d M_{r}+2 p(p-1) \int_{0}^{t} M_{r}^{p-2}\left|X_{r}\right| d r .
\end{aligned}
$$

Taking expectations and using Hölder's inequality,

$$
\begin{aligned}
\mathbb{E}\left(M_{t}^{p}\right) & =2 p(p-1) \int_{0}^{t} \mathbb{E}\left(M_{r}^{p-2}\left|X_{r}\right|\right) d r \\
& \leq 2 p(p-1) \int_{0}^{t}\left(\mathbb{E}\left(M_{r}^{p}\right)\right)^{(p-2) / p}\left(\mathbb{E}\left(\left|X_{r}\right|^{p / 2}\right)\right)^{2 / p} d r .
\end{aligned}
$$

Since $f(r)=\mathbb{E}\left(M_{r}^{p}\right)$ is continuous, the generalized Gronwall inequality (see, e.g., Bihari [7]) with $\Omega(u)=\int_{0}^{u} r^{(2-p) / 2} d r=(p / 2) u^{2 / p}$ in the notation there yields

$$
\mathbb{E}\left(M_{t}^{p}\right) \leq\left(4(p-1) \int_{0}^{t}\left(\mathbb{E}\left(\left|X_{r}\right|^{p / 2}\right)\right)^{2 / p} d r\right)^{p / 2} .
$$


Substituting the bound from equation (18) gives

$$
\mathbb{E}\left(M_{t}^{p}\right) \leq\left(4(p-1)\left(|x| t+t^{2}(c+p-2)\right)\right)^{p / 2} .
$$

Consequently,

$$
\left\|X_{t}-x\right\|_{p} \leq c t+2 \sqrt{(p-1)\left(|x| t+t^{2}(c+(p-2))\right)} .
$$

Now let $t \geq s \geq 0$. By the Markov property of $X$ at time $s$ and equations (19) and (18), we get

$$
\begin{aligned}
&\left(\mathbb{E}\left(\left|X_{t}-X_{s}\right|^{p}\right)\right)^{1 / p} \\
& \leq\left(\mathbb{E}\left(\left(c(t-s)+2 \sqrt{(p-1)\left(\left|X_{s}\right|(t-s)+(t-s)^{2}(c+p-2)\right)}\right)^{p}\right)\right)^{1 / p} \\
& \leq\left(\mathbb{E}\left(\left((t-s)(c+2 \sqrt{(p-1)(c+p-2)})+2 \sqrt{p-1} \sqrt{t-s} \sqrt{\left|X_{s}\right|}\right)^{p}\right)\right)^{1 / p} \\
& \leq(t-s)(c+2 \sqrt{(p-1)(c+p-2)}) \\
&+2 \sqrt{p-1} \sqrt{t-s} \sqrt{|x|+2 s(c+2(p / 2-1))}
\end{aligned}
$$

Corollary 34. Let $\left(X_{t}, 0 \leq t \leq 1\right)$ satisfy the hypotheses of Lemma 33. Then for every $p \in(2, \infty)$ and $\gamma \in(0,(p-2) / 2 p)$,

$$
\begin{aligned}
& \mathbb{E}\left(\left(\sup _{0 \leq s<t \leq 1} \frac{\left|X_{t}-X_{s}\right|}{|t-s|^{\gamma}}\right)^{p}\right) \\
& \quad \leq \frac{2^{\gamma p+p+1}(c+2 \sqrt{(p-1)(c+p-2)}+2 \sqrt{p-1} \sqrt{|x|+2(c+p-2)})^{p}}{\left(1-2^{\gamma+(2-p) / 2 p}\right)^{p}} .
\end{aligned}
$$

Proof. The fact that

$$
\mathbb{E}\left(\left(\sup _{0 \leq s<t \leq 1} \frac{\left|X_{t}-X_{s}\right|}{|t-s|^{\gamma}}\right)^{p}\right)<\infty
$$

is an immediate consequence of [43], Theorem I.(2.1), and the explicit bound as a function of $x, c, p$ and $\gamma$ is obtained by keeping track of the constants in the proof of that theorem.

6.3. Results for BESQ processes. The following corollary is an important special case of Corollary 34.

COROLlary 35. For $\delta \geq 1$, let $\left(X_{t}, 0 \leq t \leq 1\right)$ be a $\operatorname{BESQ}(\delta)$ process starting from 0 , and $\gamma \in(0,1 / 2)$. Then

$$
D_{\delta, \gamma}=\sup _{0 \leq s<t \leq 1} \frac{\left|X_{t}-X_{s}\right|}{|t-s|^{\gamma}}<\infty \quad \text { a.s. }
$$

with moments of all orders. 
COROLlary 36. For $\delta \geq 1$, let $Z$ be a standard $\operatorname{BESQ}(\delta)$ bridge from 0 to 0 or equivalently a BESQ $(4-\delta)$ excursion, and let $\gamma \in(0,1 / 2)$. Then

$$
D_{\gamma}^{*}=\sup _{0 \leq s<t \leq 1} \frac{\left|Z_{t}-Z_{s}\right|}{|t-s|^{\gamma}}<\infty \quad \text { a.s. }
$$

with moments of all orders.

Proof. The equivalence of $\operatorname{BESQ}(\delta)$ bridges and $\operatorname{BESQ}(4-\delta)$ excursions was noted in [41], Remark (5.8)(i). By [43], Exercises XI.(3.6)-(3.7), the process $\left(Z_{1-t}, 0 \leq t \leq 1\right)$ has the same distribution as $Z$ and we can write $Z_{u}=u^{2} X_{1 / u-1}$ for a $\operatorname{BESQ}(\delta)$ process $X$ starting from 0 . For $1 / 2 \leq s<t \leq 1$, we then obtain by the previous corollary

$$
\begin{aligned}
\left|Z_{t}-Z_{s}\right| & =\left|t^{2} X_{1 / t-1}-s^{2} X_{1 / s-1}\right| \\
& \leq\left|X_{1 / t-1}-X_{1 / s-1}\right|+\left(t^{2}-s^{2}\right) X_{1 / s-1} \\
& \leq 4^{\gamma} D_{\delta, \gamma}|t-s|^{\gamma}+2|t-s|^{\gamma} \bar{X},
\end{aligned}
$$

where $\bar{X}=\sup _{0 \leq r \leq 1} X_{r}$. Similarly, we can write $Z_{u}=(1-u)^{2} X_{1 /(1-u)-1}$ for another (dependent) $\operatorname{BESQ}(\delta)$ process $X$ starting from 0 , and for $0 \leq s<t \leq 1 / 2$, we obtain $\left|Z_{t}-Z_{s}\right| \leq\left(4^{\gamma} \widetilde{D}_{\delta, \gamma}+2 \bar{X}\right)|t-s|^{\gamma}$. Finally, for $0 \leq s<1 / 2<t \leq t$, the triangular inequality yields the required bound so that

$$
D_{\gamma}^{*} \leq 4^{\gamma} D_{\delta, \gamma}+2 \bar{X}+4^{\gamma} \widetilde{D}_{\delta, \gamma}+2 \bar{X}
$$

has moments of all orders.

6.4. BESQ-marked stable processes and their local times. If $X$ has Laplace exponent $\psi(\eta)=\eta^{1+\alpha}$, then $X_{a}(t)=X_{a t}, t \geq 0$, has Laplace exponent $\psi_{a}(\eta)=$ $a \eta^{1+\alpha}$. From the occupation density formula, we see easily that

$$
\ell_{a}^{y}(t)=\frac{1}{a} \ell^{y}(a t) \quad \text { and } \quad \tau_{a}^{y}(s)=\frac{1}{a} \tau^{y}(a s) .
$$

Leaving marks unscaled, $m_{h, a}(y, t)=m_{h}(y, a t)$, and Theorem 1 holds with $c_{a}=$ $a c$, Corollary 2 with $c_{a}^{\circ}=c^{\circ} / a$.

Specifically, choosing $q=1$ and $\kappa_{1}$-scaled $\operatorname{BESQ}(-2 \alpha)$ excursions, we can calculate

$$
\mathbb{E}\left(Z_{U}^{\alpha}\right)=\frac{2^{\alpha} \Gamma(1+\alpha)}{1+\alpha} \Rightarrow c_{a}=a \frac{\Gamma(1+\alpha)}{\Gamma(1-\alpha)} 2^{\alpha}
$$

Apart from $a=1$, there are various other natural choices that keep constants in certain formulas simple. For the following, we choose $c_{a}=1 / \Gamma(1-\alpha)$, so that Proposition 8 yields Laplace exponent $\Theta_{a}(\xi)=\xi^{\alpha}$. This corresponds to setting $a=1 / \Gamma(1+\alpha) 2^{\alpha}$. For $\alpha=1 / 2$, these are $a=\sqrt{2 / \pi}$ and $c_{a}=1 / \sqrt{\pi}$. For illustration and ease of reference in [19] and future work, let us restate Theorem 1 in this special case. 
THEOREM 37. Let $\alpha \in(0,1)$ and $a=1 / \Gamma(1+\alpha) 2^{\alpha}$. For $a \operatorname{BESQ}(-2 \alpha)$ marked stable process $X_{a}$ with Laplace exponent $\psi_{a}(\eta)=a \eta^{1+\alpha}$, we have almost surely

$\lim _{h \downarrow 0} \sup _{0 \leq t \leq T} \sup _{y \in \mathbb{R}}\left|\Gamma(1-\alpha) h^{\alpha} m_{h, a}(y, t)-\ell_{a}^{y}(t)\right|=0, \quad$ for all $T>0$,

where local times $\ell_{a}^{y}(t)$ and mass counts $m_{h, a}(y, t)$ are associated with $X_{a}$ for $y \in \mathbb{R}, t \geq 0, h>0$.

PROOF. To apply Theorem 1, let us first note that the Hölder constant of a $\operatorname{BESQ}(-2 \alpha)$ excursion has moments of all orders by Corollary 36 . Then, for our choice $a=1 / \Gamma(1+\alpha) 2^{\alpha}$ and $c_{a}=a c$, we find that

$$
\left|\Gamma(1-\alpha) h^{\alpha} m_{h, a}(y, t)-\ell_{a}^{y}(t)\right|=\frac{1}{a}\left|\frac{h^{\alpha}}{c} m_{h}(y, a t)-\ell^{y}(a t)\right|,
$$

and the application of Theorem 1 yields that the relevant suprema tend to 0 as $h \downarrow 0$.

\section{REFERENCES}

[1] BARLOW, M. T. (1988). Necessary and sufficient conditions for the continuity of local time of Lévy processes. Ann. Probab. 16 1389-1427. MR0958195

[2] Barlow, M. T., Perkins, E. A. and Taylor, S. J. (1986). The behaviour and construction of local times for Lévy processes. In Seminar on Stochastic Processes, 1984 (Evanston, Ill., 1984). Progr. Probab. Statist. 9 23-54. Birkhäuser, Boston, MA. MR0896720

[3] Barlow, M. T., Perkins, E. A. and Taylor, S. J. (1986). Two uniform intrinsic constructions for the local time of a class of Lévy processes. Illinois J. Math. 30 19-65. MR0822383

[4] Bertoin, J. (1996). Lévy Processes. Cambridge Tracts in Mathematics 121. Cambridge Univ. Press, Cambridge. MR1406564

[5] Bertoin, J. (1996). On the first exit time of a completely asymmetric stable process from a finite interval. Bull. Lond. Math. Soc. 28 514-520. MR1396154

[6] Bertoin, J. (1997). Exponential decay and ergodicity of completely asymmetric Lévy processes in a finite interval. Ann. Appl. Probab. 7 156-169. MR1428754

[7] BIHARI, I. (1956). A generalization of a lemma of Bellman and its application to uniqueness problems of differential equations. Acta Math. Hungar. 7 81-94.

[8] Blumenthal, R. M. and Getoor, R. K. (1968). Markov Processes and Potential Theory. Pure and Applied Mathematics 29 Academic Press, New York. MR0264757

[9] BoYLAn, E. S. (1964). Local times for a class of Markoff processes. Illinois J. Math. 8 19-39. MR0158434

[10] Caballero, M. E. and Chaumont, L. (2006). Conditioned stable Lévy processes and the Lamperti representation. J. Appl. Probab. 43 967-983.

[11] Chacon, R. V., Le Jan, Y., Perkins, E. and Taylor, S. J. (1981). Generalised arc length for Brownian motion and Lévy processes. Z. Wahrsch. Verw. Gebiete 57 197-211. MR0626815

[12] CsÖRGŐ, M. and RÉvÉSz, P. (1986). Mesure du voisinage and occupation density. Probab. Theory Related Fields $\mathbf{7 3}$ 211-226. MR0855223 
[13] Delaporte, C. (2013). Lévy processes with marked jumps II: Application to a population model with mutations at birth. Preprint. Available at arXiv:1305.6491 [math.PR].

[14] Delaporte, C. (2015). Lévy processes with marked jumps I: Limit theorems. J. Theoret. Probab. 28 1468-1499. MR3422939

[15] Dembo, A. and Zeitouni, O. (2010). Large Deviations Techniques and Applications. Stochastic Modelling and Applied Probability 38. Springer, Berlin. Corrected reprint of the second (1998) edition. MR2571413

[16] Doney, R. A. (2007). Fluctuation Theory for Lévy Processes. Lecture Notes in Math. 1897. Springer, Berlin. MR2320889

[17] EthieR, S. N. and KURTZ, T. G. (1981). The infinitely-many-neutral-alleles diffusion model. Adv. in Appl. Probab. 13 429-452. MR0615945

[18] Ethier, S. N. and Kurtz, T. G. (1986). Markov Processes. Characterization and Convergence. Wiley, New York. MR0838085

[19] Forman, N., Pal, S., Rizzolo, D. and Winkel, M. (2017). Diffusions on a space of interval partitions with Poisson-Dirichlet stationary distributions. Available at arXiv:1609.06706v2 [math.PR].

[20] Getoor, R. K. and Sharpe, M. J. (1981). Two results on dual excursions. In Seminar on Stochastic Processes, 1981 (Evanston, Ill., 1981). Progr. Prob. Statist. $131-52$. Birkhäuser, Boston, MA. MR0647780

[21] Gnedin, A., Pitman, J. and Yor, M. (2006). Asymptotic laws for compositions derived from transformed subordinators. Ann. Probab. 34 468-492. MR2223948

[22] GöIng-Jaeschke, A. and Yor, M. (2003). A survey and some generalisations of Bessel processes. Bernoulli 9 313-349.

[23] Hutzenthaler, M., Jentzen, A. and Noll, M. (2014). Strong convergence rates and temporal regularity for Cox-Ingersoll-Ross processes and Bessel processes with accessible boundaries. ArXiv preprint. Available at arXiv:1403.6385.

[24] Jagers, P. (1974). Convergence of general branching processes and functionals thereof. J. Appl. Probab. 471-478.

[25] Jagers, P. (1975). Branching Processes with Biological Applications. Wiley, New York. MR0488341

[26] Kallenberg, O. (2002). Foundations of Modern Probability, 2nd ed. Springer, New York. MR1876169

[27] Khoshnevisan, D. (1994). Exact rates of convergence to Brownian local time. Ann. Probab. 22 1295-1330. MR1303646

[28] Kyprianou, A. E. (2006). Introductory Lectures on Fluctuations of Lévy Processes with Applications. Springer, Berlin. MR2250061

[29] Kyprianou, A. E., Pardo, J. C. and Watson, A. R. (2014). Hitting distributions of $\alpha$-stable processes via path censoring and self-similarity. Ann. Probab. 42 398-430. MR3161489

[30] LAMBert, A. (2000). Completely asymmetric Lévy processes confined in a finite interval. Ann. Inst. Henri Poincaré Probab. Stat. 36 251-274. MR1751660

[31] LAmbert, A. (2010). The contour of splitting trees is a Lévy process. Ann. Probab. 38 348395. MR2599603

[32] Lambert, A. and Simatos, F. (2013). Asymptotic behavior of local times of compound Poisson processes with drift in the infinite variance case. J. Theoret. Probab. 28 41-91.

[33] Lambert, A. and Uribe Bravo, G. (2016). Totally ordered measured trees and splitting trees with infinite variation. ArXiv preprint. Available at arXiv:1607.02114.

[34] Pardo, J. C., Pérez, J. L. and Rivero, V. M. (2015). The excursion measure away from zero for spectrally negative Lévy processes. ArXiv preprint. Available at arXiv:1507.05225. 
[35] Perkins, E. (1981). A global intrinsic characterization of Brownian local time. Ann. Probab. 9 800-817. MR0628874

[36] Perman, M. (1996). An excursion approach to Ray-Knight theorems for perturbed Brownian motion. Stochastic Process. Appl. 63 67-74.

[37] Petrov, L. A. (2009). A two-parameter family of infinite-dimensional diffusions on the Kingman simplex. Funktsional. Anal. i Prilozhen. 43 45-66. MR2596654

[38] Pistorius, M. R. (2003). On doubly reflected completely asymmetric Lévy processes. Stochastic Process. Appl. 107 131-143. MR1995924

[39] Pistorius, M. R. (2004). On exit and ergodicity of the spectrally one-sided Lévy process reflected at its infimum. J. Theoret. Probab. 17 183-220. MR2054585

[40] Pitman, J. (2006). Combinatorial Stochastic Processes. Lecture Notes in Math. 1875. Springer, Berlin. MR2245368

[41] Pitman, J. and Yor, M. (1982). A decomposition of Bessel bridges. Z. Wahrsch. Verw. Gebiete 59 425-457. MR0656509

[42] Pitman, J. and Yor, M. (1997). The two-parameter Poisson-Dirichlet distribution derived from a stable subordinator. Ann. Probab. 25 855-900. MR1434129

[43] RevuZ, D. and Yor, M. (1999). Continuous Martingales and Brownian Motion, 3rd ed. Grundlehren der Mathematischen Wissenschaften [Fundamental Principles of Mathematical Sciences] 293. Springer, Berlin. MR1725357

[44] Rogers, L. C. G. and Williams, D. (2000). Diffusions, Markov Processes, and Martingales. Vol. 2. Itô Calculus, Cambridge Univ. Press, Cambridge. MR1780932

[45] Ruggiero, M., Walker, S. G. and Favaro, S. (2013). Alpha-diversity processes and normalized inverse-Gaussian diffusions. Ann. Appl. Probab. 23 386-425. MR3059239

[46] Simon, T. (2011). Hitting densities for spectrally positive stable processes. Stochastics 83 203-214.

N. FORMAN

S. PAL

DePARTMENT OF MATHEMATICS UNIVERSITY OF WASHINGTON

SEATTLE, WASHINGTON 98195 USA

E-MAIL: noah.forman@gmail.com soumikpal@gmail.com
D. RizZOLO

Department of Mathematical SCIEnCES

UNIVERSITY OF DELAWARE

NEWARK, DELAWARE 19716

USA

E-MAIL: drizzolo@udel.edu

\author{
M. WINKEL \\ DEPARTMENT OF STATISTICS \\ UNIVERSITY OF OXFORD \\ 24-29 ST GILES' \\ OXFORD OX1 3LB \\ UNITED KINGDOM \\ E-MAIL: winkel@stats.ox.ac.uk
}

